\title{
Design and Development of a Mechanical Linear Scanning Device for the Three-Dimensional Ultrasound Imaging System
}

\author{
Tita Karlita ${ }^{1,3}$, Eko Mulyanto Yuniarno ${ }^{1,2}$, I Ketut Eddy Purnama ${ }^{1,2}$, \\ and Mauridhi Hery Purnomo ${ }^{1,2}$ \\ ${ }^{1}$ Institut Teknologi Sepuluh Nopember, Department of Electrical Engineering \\ ${ }^{2}$ Institut Teknologi Sepuluh Nopember, Department of Computer Engineering \\ ${ }^{3}$ Electronics Engineering Polytechnic Institute of Surabaya, \\ Department of Information Technology, INDONESIA \\ tita16@mhs.ee.its.ac.id, tita@pens.ac.id, ekomulyanto@ee.its.ac.id, \\ ketut@ee.its.ac.id, hery@ee.its.ac.id
}

\begin{abstract}
Three-dimensional reconstruction from a set of a two-dimensional ultrasound image is useful for visualizing anatomy structures of an internal organ. This paper presents a mechanical linear scanning device for a three-dimensional ultrasound imaging system to acquire sequences of two-dimensional ultrasound image along with its position and orientation in three-dimensional space. The device included a linear sliding track driven by a stepper motor with a dedicated housing of a probe, a water tank with an object holder inside, and software installed on a standard computer. A mechanical sliding track enabled the two-dimensional conventional ultrasound probe moved in a regular linear manner. The mechanical linear scanning allowed fixed and predefined translation steps in z-axis direction such that rotation error could be avoided. In addition, mathematical formulations were presented to relocate every pixel's location of the ultrasound image to the reconstruction volume in three-dimensional space. In the experiments, the device's installation in a real environment was demonstrated, and the translation motion in space was investigated. Evaluation of the scanning results using the proposed device was performed using a bone as a phantom. The experimental result showed that the proposed device produced high quality scanning results and created high quality bone surface. Thus, it can be expected would be useful to the three-dimension reconstruction applications.
\end{abstract}

Keywords: mechanical linear scanning, three-dimensional reconstruction, ultrasound imaging system.

\section{Introduction}

Medical imaging visualizes a particular internal anatomical region of a human body in twodimensional (2D) images. These images provide limited information about the shape and structure of the human internal organ because they are presented in the form of images. Therefore it is difficult to analyze the complex shapes and structures of the organs and get its accurate morphometric information quantitatively [1][2]. The medical experts must check these 2D images slice by slice to the end to analyse the exact position of the problem within their minds. Moreover, this procedure is time consuming, inefficient, and depends on the experience and expertise of the experts in analyzing problems. This fact has highlighted the need to reconstruct three-dimensional (3D) anatomical structure from a set of 2D images so that the measurements of organ dimensions could be investigated accurately [3][4]. This mechanism extends the narrow field of view of conventional 2D ultrasound.

Ultrasound is often used as a technique of imaging internal structures of a human body such as soft tissues and bone since it is non-invasive, no radiation exposure, relatively inexpensive, compact, and provide real-time data acquisition [5][6]. It has been a safe and effective tool for internal body imaging than MRI and CT-scan. It also has been widely used in many medical centers for clinical purposes. The major drawback of conventional two-dimensional ultrasound is its low image quality and lacking in determining quantitative and accurate volume information since it has limitations with spatial imaging and precise volume measurement [1][6][7].

Received: March $14^{\text {th }} \cdot 2018$. Accepted: June $27^{\text {th }}, 2019$

DOI: 10.15676/ijeei.2019.11.2.4 
Over the past three decades, various 3D imaging systems have been proposed for the construction and visualization of various internal organs. Referring to Fenster et al. [1], Mercier et al. [5], and with the addition of methods according to recent developments in the 3D ultrasound imaging system, there are several tracking techniques and scanning methods used to acquire $2 \mathrm{D}$ ultrasound images with the primary goal of creating an accurate $3 \mathrm{D}$ volume. These $2 \mathrm{D}$ ultrasound images are also known as B-scans. There are two tracking approaches to estimate B-scans relative position and orientation information in 3D space, i.e., sensor-based and sensorless tracking approach. In the sensor-based tracking approach system, an ultrasound probe is equipped with a position sensor while the sensorless tracking approach does not use a position sensor. Here, a position sensor is required to identify probes motions during the scanning. Generally, the position sensors used in the freehand technique are electromagnetic [3][8], optical [9][10-14], and acoustic. Electromagnetic and optical are the most popular approach. However, scanning using a position sensor is time-consuming because once the user changes the position of the sensor, the calibration process has to be repeated [6].

In freehand technique with a position sensor, a $2 \mathrm{D}$ conventional ultrasound probe moves freely in unconstrained movement over the body part to obtain irregularly and non-homogenous spaced B-scans slices. A position sensor will report probes position and orientation simultaneously. In the freehand technique, an ultrasound probe is freely controlled by users. It produces irregularly spaced B-scan images along an random path [12]. Freehand 3D ultrasound is useful for scanning large objects or scanning around a curved surface to obtain its volume. Nevertheless, the physician has to practice how to move the ultrasound probe in steady and evenly motion to collect regular and densely sampled data sets [8]. Other characteristics of freehand scanning are slow acquisition process and the possibility of image deviation appearance caused by different probe pressure when contact with organ [13].

In sensorless tracking approach, the estimation of position and orientation of B-scans are determined without any position sensor. The estimation of position and orientation of the ultrasound probe in 3D was approximated by using speckle decorrelation [6][13][14], linear regression [15], without any position tracking [16] or using articulated arm (robotized) [17][18]. Commonly, the assumption of its position and orientation uses image correlation or linear regression approach. Sensorless tracking approach using image correlation was based on the speckle decorrelation phenomenon. The result of the study showed that two images that are obtained from the same position have the same speckle pattern so that there will be no decorrelation. Therefore, the gap between the two image pairs can be determined. The fact that two image pairs are not always parallel to each other make this system is complicated [1]. Nevertheless, because each B-scan is arranged based on the previous B-scan, cumulative errors and drift are inevitable [19]. Moreover, Li stated that analysis based only on speckle correlation was not enough to solve the problem of freehand scanning [20]. In comparison with Prager's [15] research of sensorless approach, their results are promising, but their accuracy is still far from tracked probes [5]. Sato et al. [7] reconstructed 3D ultrasound phantom to analyzed refractive artifacts to obtain a better understanding of the effect of sound refraction in ultrasound images. $3 \mathrm{D}$ volume was reconstructed from sequences of $2 \mathrm{D}$ images which were taken by using conventional freehand ultrasound. The ultrasound probe was slid slowly and steadily by hand and was perpendicular to the phantom with substantial care to minimize translational or rotational movement.

Commonly, 3D ultrasound imaging systems use freehand ultrasound which is equipped with a position sensor, i.e., optical and electromagnetic sensor. This system has 6-Degree of Freedom (DoF) so that the ultrasound probe can move freely in an unconstrained manner by hand over the anatomy in several directions during the data acquisition process to obtain sequences of $2 \mathrm{D}$ ultrasound images. The nature of 6-DoF produces a plane that is not sequential, non-uniformly spaced B-scans, and there is a lot of empty space. This causes more effort to fill the empty space using a hole filling method that requires more computation time [4][6][12]. Therefore, it depends on the physician to move a conventional probe over the anatomy [8]. Moreover, it is well recognized that the reading of a position sensor is not always precise. A position sensor is 
susceptible to small utters that causes measurement error happen. There are two kinds of error movement in the 6-DoF system, i.e., three types of translational errors and three types of rotational errors. The rotational error is resulting in larger inaccuracies than the translational error in the reconstruction process [12]. The problem is the appearance of visible artifacts in images as a result of small error measurement in the position sensor reading [12][13].

The utilization of position sensors in 3D medical imaging systems needs special environment setting to ensure the correctness reading of their position and orientation estimation. Data sensing using optical sensor requires an unobstructed view of the marker mounted to a probe and tracking cameras. On the other hand, the use of an electromagnetic sensor is challenging because it is affected by two-metal physical related phenomena. Various metallic or ferromagnetic materials around in the field can influence the performance of electromagnetic tracking systems [5][6][11]. The operator must stay inside the working region of the sensor and maintain a magnetic field sensor away from electromagnetic interference. These limitations have motivated researchers to develop efficient, convenient, and reliable methods for 3D ultrasound reconstruction. Huang [12][21] stated that sample data acquisition is a crucial stage. He recommended that the scanning technique should be limited in one or multiple sweeps and the probe movement is thereabout linear along with only single direction [12]. To obtain 3D volume with high accuracy, Gee suggested that a steady, stable speed and single sweep of the probe in evenly gaps was favored rather than that in an arbitrary manner [22].

Compared to the number of the sensor-based and sensorless study of 3D ultrasound imaging systems, studies of sensorless approaches still have relatively low numbers. This fact opens a wide opportunity to explore more about developing sensorless 3D ultrasound imaging systems. This paper aims to design and develop a mechanical linear scanning device for $3 \mathrm{D}$ ultrasound imaging system with a reduced DoF. The linear scanning system driven by a stepper motor has a probe holder to attach a conventional ultrasound probe. A stepper motor controls the motion of the probe to enable 1-DoF motions with no rotation. Our proposed 3D ultrasound imaging system was tested to scan a bone as a phantom model and the motion of the linear tracker was investigated as well. The system design and the transformation formula to relocate every pixel's location in B-scans to the reconstruction volume in the $3 \mathrm{D}$ coordinate system are first presented. Finally, the demonstration of the device in a real environment is presented and the scanning results are evaluated.

This article is organized as follows: Previous research relevant to sensorless 3D ultrasound imaging systems are summarized in Sec. 2. Section 3 describes the framework of our 3D reconstruction using a mechanical linear 3D ultrasound imaging system. Begin with an explanation of the 3D reconstruction global framework. It was followed by the description of the design, specifications, and development of a 3D ultrasound imaging system device. Then, our device is analyzed on how to create volume reconstruction. The number of coordinate systems location, its transformation, and its physical relationships are identified. Finally, our notation and the mathematical 3D reconstruction formulation are formally stated. In addition, parameters to be solved in the probe calibration process are presented. Moreover, the object scanning result and its generated point clouds are evaluated. Section 4 presents the experimental result of the proposed system. Discussion of these works is presented in Sec. 5.

\section{Sensorless 3D Ultrasound Imaging System}

In this section, the previously published literature on the development of sensorless 3D ultrasound imaging systems is presented. In the sensorless approach, there are some techniques used to estimate the 3D position and orientation of a probe in space, i.e., using speckle decorrelation[6][13][14], linear regression [15], without any position tracking [16], or using articulated arm (robotized) [17][18].

Because the use of the position sensor was impractical and can be complicated for the operator, Prager et al. [15] presented an alternative approach based on linear regression of the echo-envelope intensity signal to specify position and orientation of B-scans. The theory was based on the theory that the back-scattered ultrasound signal contains only fully developed 
speckle. Image intensity in a continuous scan and the distance between them was used to create a model to predict the expected intensity in one scan. The model was based on Bayes's theorem. The Levenberg-Marquardt algorithm was used to align the 3D locations. However, Mercier et al. stated that although Prager's research results are encouraging, the accuracy of sensorless approach is far compared to sensor-based probes [5].

Another approach of sensorless freehand 3D ultrasound reconstruction algorithm was using speckle decorrelation. Chang et al. [14] proposed a new positioning system for 3D ultrasound without any positioning sensor. They combined image registration method and speckle decorrelation algorithm to precisely determine consecutive ultrasonic images positions which needed only a reference image to match it. Housden et al. [13] utilized speckle decorrelation to approximate the geometric orientation of B-scans which were obtained using freehand ultrasound system. Their method was assessed using sensor-based datasets of a beef phantom. They resumed that small miss positioning of the B-scans results in breaks in the sensor-based slices, whereas it was smoother in the sensorless slices. To obtain position and orientation information of the B-scans, Gao et al. [6] did not need any position sensing device. They referred to adaptive speckle decorrelation tracking to obtain spatial information of the B-scans. Speckle decorrelation was used to specify the relative position and orientation between two adjacent Bscan images. A laptop equipped with a conventional linear ultrasound probe was used to obtain B-scan images. A software program was developed for capturing the B-scans during a freehand scanning. Wi-Fi connection was needed to send B-scans to a remote workstation in real-time. Nevertheless, Li stated that it was impossible to determine 3D spatial information of freehand scanning based only speckle correlation analysis in a real environment [20].

To acquire set of B-scans using the freehand scanning techniques with no position sensing approach, ultrasound probe should be moved carefully and steadily in the uniform spaced over the patient body controlled by user's hand in the linear or constant angular motion. The 3D volume reconstruction was obtained by assuming a predefined scanning geometry. Gee et al. [16] performed a 3D ultrasound probe calibration without any position sensor. The ultrasound probe was attached on to a manufactured mechanical instrument which allows height adjustment. The relative position and orientation of the probe when captured a set of co-planar wires strung as a calibration phantom can be adjusted and measured. Nevertheless, Fenster studied that any measurements cannot be determined since this methodology cannot guarantee that the 3D result was geometrically accurate [1].

To obtain parallel and evenly spaced ultrasound cross section of femur ultrasound images, Torres et al. [18] used a robotized ultrasound probe. An anthropomorphic arm robot with a 5DoF movement was used to acquire B-scans so that the position and orientation of B-scans were known precisely. Since the 3D imaging system has 5-DoF, each B-scan was processed with respect to translation and rotation displacement according to the homogeneous transformation matrix. A 5-DoF parallel robot was developed by Lessard et al. [17] to tracked 3D ultrasound examinations of human peripheral arteries. System's direct and inverse kinematics was solved by using a geometrical approach. Merouche et al. [23] designed a robotic ultrasound system to scan and create a 3D volume of a sequence of the lower limb arterial tree between the groin and the knee. The prototype was composed of a 6-DoF industrial F3 articulated robot arm, an open platform ultrasound scanner, and a 128-element linear array probe.

\section{Materials and Methods}

The position of our proposed framework of 3D reconstruction using a mechanical linear 3D ultrasound imaging system can be seen in Figure 1. It begins with the design and development of a device for object scanning to obtain a sequence of ultrasound images along with its position and orientation in 3D space. Then, device calibration needs to be performed to get the correspondence between every pixels position in an ultrasound image and the $3 \mathrm{D}$ volume. In the data acquisition process, the object was scanned many times in different positions to obtain several sets of bone ultrasound images. The image segmentation process is then conducted to obtain object outer contours. Then, a set of object outer contours is arranged by referring to its 
spatial coordinates in 3D to build an object outer surface. When the object being scanned is a hard object such as a bone, the ultrasound signal can only capture a portion of the surface. Therefore, an alignment process is needed to align several object surfaces until a whole object surface is formed. To analyze the generated 3D object, 3D distance measurements are conducted to assess the object outer surface against its reference model as ground truth.

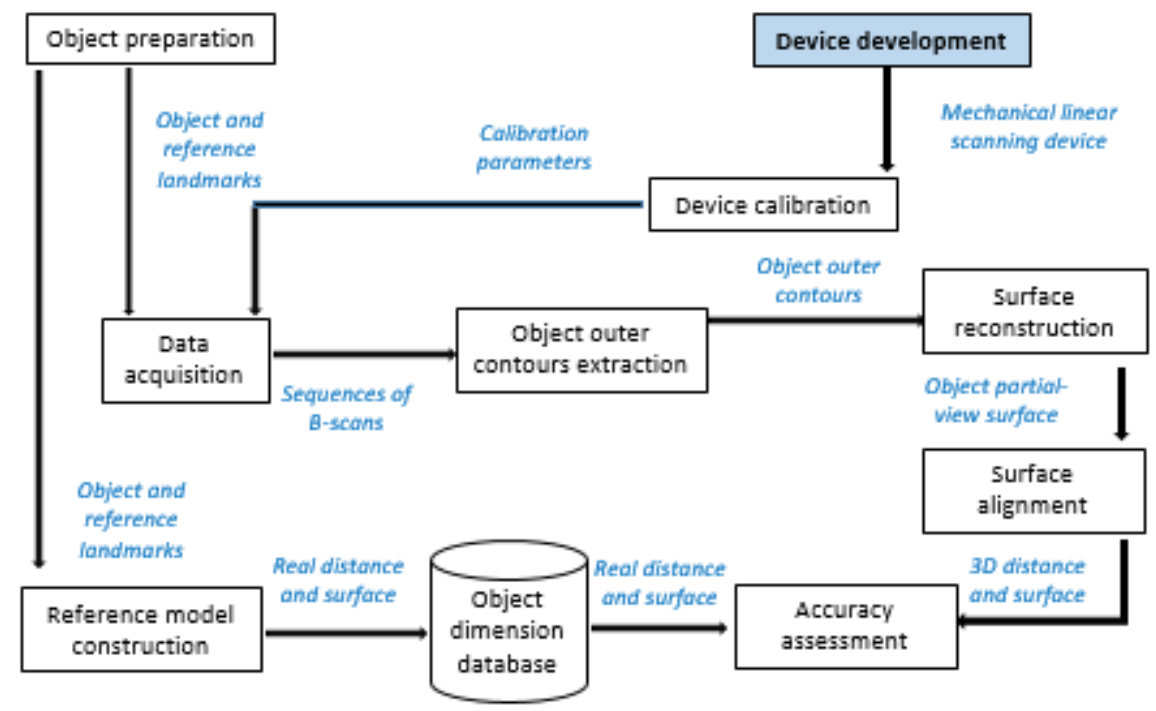

Figure 1. Position of the device development process in the diagram of the framework of 3D reconstruction using a mechanical linear 3D ultrasound imaging system.

The focus of this study develops a scanning device called a mechanical linear 3D ultrasound imaging system as a part of the framework of 3D reconstruction using a mechanical linear 3D ultrasound imaging system. Methods explanation of the device development in more detailed can be found in the next sub-chapters.

\section{A. Design and Development of a Mechanical Linear Scanning Device}

In this paper, a mechanical linear scanning device for 3D ultrasound imaging system was designed and developed. The schematic diagram of the system is illustrated in Figure 2. The device was comprised of three main subsystems: (i) a linear sliding track driven by a stepper motor with a dedicated housing of an ultrasound probe and a water bath with a bone or object holder inside, (ii) a beamformers as a general purpose ultrasonic pulse-receiver which transferring ultrasound data to a standard computer, and (iii) a standard computer to control the movement of ultrasound probe using a stepper motor and to visualize and collect the B-scans. In this system, a TELEMED Ultrasound medical systems Linear Transducer L15-7L40H-5 with frequency ranges of 7.0-15.0 MHz and $39 \mathrm{~mm}$ field of view to obtain a series of localized ultrasound images was used. The beamformers were LS 64 SmartUs OEM, UAB model SmartUs EXT-1M series. It allowed integration between the ultrasound probe and common computer and was used to achieve high-quality ultrasonic images.

To move the conventional 2D ultrasound probe in a linear direction (1-DoF), a software that was running in a common computer was developed. There were two kinds of software's developed. The first was a microcontroller software; an Arduino board was programmed to activate a stepper motor and control the ultrasound probe's motion in a specified user preferred distance. The second was an ultrasound software; it captured and saved b-mode ultrasound images automatically when the ultrasound probe moves in the linear track. 


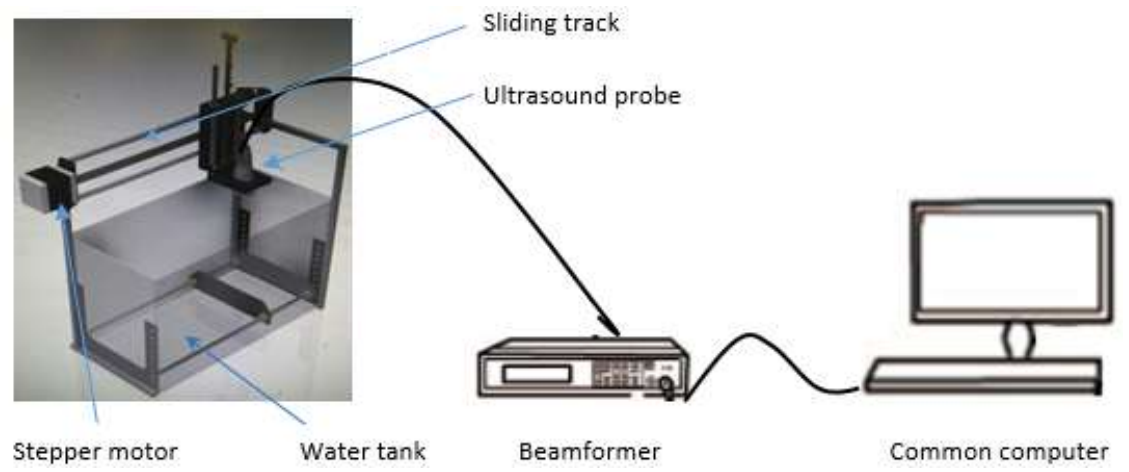

Figure 2. A schematic diagram of the mechanical linear 3D ultrasound imaging system device. The device consists of three main parts: a water tank with a linear track, a beamformer, and a common computer.

The workflow of the device, starting from moving the ultrasound probe until saving the images is shown in Figure 3. The workflow diagram consisted of three parts, (i) the common computer which had two software's (ultrasound controller and microcontroller), (ii) an ultrasound part which had a beamformers and an ultrasound probe, and (iii) a linear track part which had Arduino board, a stepper motor, and a probe holder. To move the conventional 2D ultrasound probe in a linear direction (one Degree of Freedom (1-DoF)), a software that is running in a common computer was developed. There were two kind of software's developed. The first was a microcontroller software, an Arduino board was programmed to activate a stepper motor and control the ultrasound probe's motion in a specified user preferred distance. The second was an ultrasound software, it captures and saves b-mode ultrasound images automatically when the ultrasound probe moves in the linear track. To run the system, it started with setting the distance per step to the microcontroller software. Then, the software sent the instruction to the Arduino board to operate the stepper motor so that the probe holder moved in the sliding track. Just after the probe holder moved, the video card captured the b-mode image produced by beamformers. The stream of the b-mode image sequence was collected by the ultrasound controller software and then stored in the disk systematically.

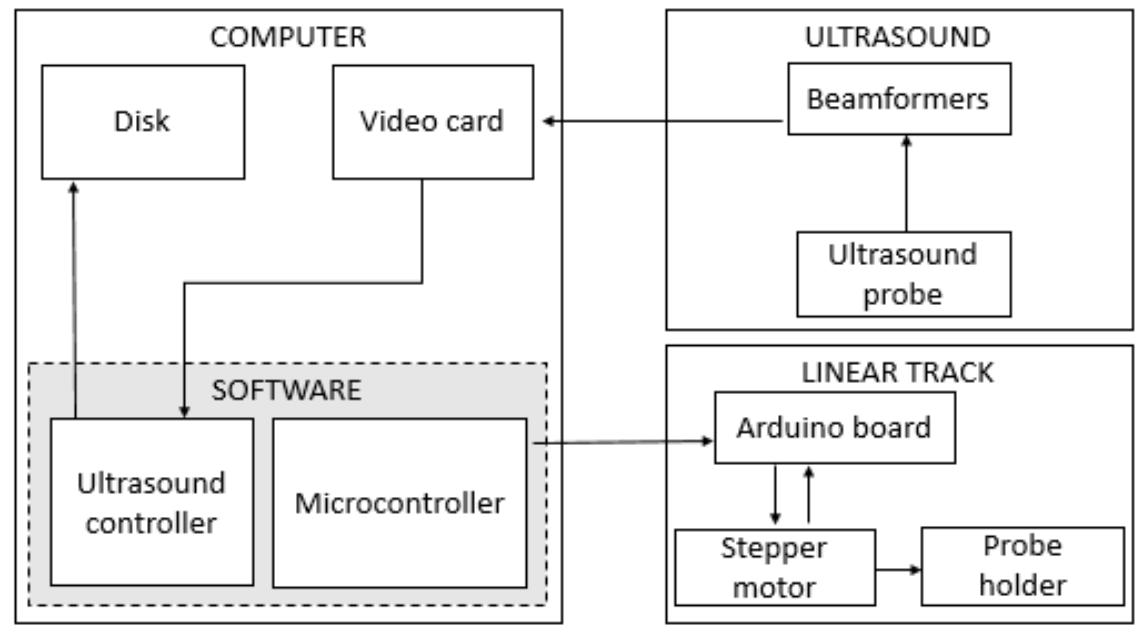

Figure 3. The workflow diagram of the ultrasound probe motion in the linear track and the bmodes capture process.

The sliding track with probe holder fixedly attached was designed as shown in Figure 4. The probe holder was able to move linearly along the path in $\mathrm{z}$-axes directions with no rotation, and 
it was driven by a stepper motor. The components that were used to run the probe in the sliding track are an Arduino Nano, a Driver Stepper ULN2004, an adaptor 12 Volt 3 Ampere, and a stepper motor torsion $4 \mathrm{~kg} 1.8$ degree. In real applications, the scanning range of the probe could be adequately controlled by users to obtain best B-scan since the height of the probe holder could be easily vertically adjusted within a range of $0-300 \mathrm{~mm}$. The motion of the probe was step by step in a predefined fix distance. The amount of steps per image capture was controlled by the user. The lesser the step, the denser the B-scans sequence would be. In every step, the user was able to collect B-scans sequentially and obtained sequences of B-scans. When the ultrasound probe was moved by the user, the B-scans in an 8-bit gray image and their position in z-axis direction were collected by the computer.

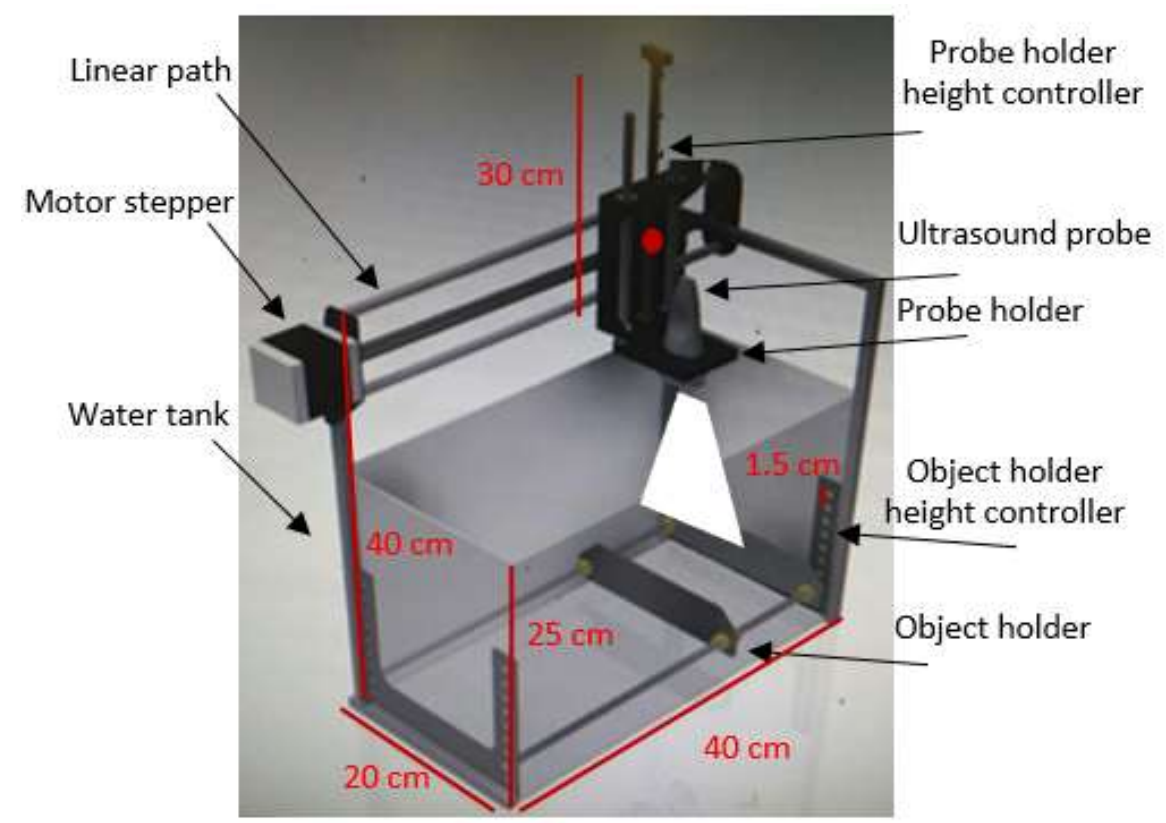

Figure 4. The Design of a mechanical linear sliding track with a probe holder, and a water tank with an object holder. The dimension of each parts were printed in red color. Each parts are named in black color.

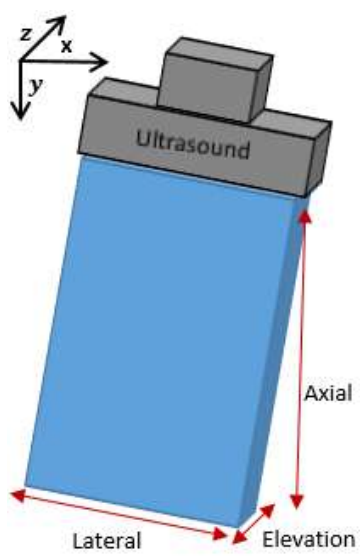

Figure 5. Illustration of a 2D linear ultrasound probe used in the proposed 3D imaging system with the axial, lateral and elevation direction 
Figure 5 illustrates the ultrasound probe lateral, axial, and elevation direction in space. In order to acquire best images, the ultrasound probe position was attempted to be perpendicular to the bone, moving in step by step over the sliding track in the elevation direction along the sliding track. The direction of the $y$-axis was in the vertical direction perpendicular to the ground, and the direction of the $\mathrm{z}$-axis was in elevation direction same as the moving direction of the probe. The illustration of a water tank and mechanical linear sliding track can be shown in Figure 4. In our design, the outer dimensions of the water tank were about $40 \mathrm{~cm}$ of length, $20 \mathrm{~cm}$ of width, and $25 \mathrm{~cm}$ of height. The ultrasound probe was installed in a fix location and orientation at the probe holder and was maintained as much as possible to be perpendicular with an object in the water tank.

With the consideration, that body parts had various geometric shapes and its surface was not always at, the probe might not press homogeneously during scanning. Thus, the $3 \mathrm{D}$ volume might not be properly constructed. As a solution, the object was submerged in the water tank, and the ultrasound probe was non-contact with it. There was an object holder which had two at irons to pin object inside the water tank as illustrated in Figure 4. Both irons can be shifted in either direction to adjust to the size of the object by screwing the bolts. Inside the water tank, there was an object holder height controller in the form of eight vertically arranged holes. The distance between the holes was $1.5 \mathrm{~cm}$. Therefore, the height of the bone holder could be easily adjusted according to objects specific size within a range of $0-120 \mathrm{~mm}$.

A common computer running with Windows 8.0 operating system 64-bit was installed with TELEMED Echo Wave II Software and Drivers Package that was responsible for ultrasound image visualization, data acquisition, and save a sequence of B-scans. Echo Wave II is a PCbased software-driven platform. It allows us to control the ultrasound system from personal computers. It has an interface like in many ultrasound scanners and has selectable and customizable user settings, such as saving and printing modes, outdoor applications, measurements and calculations, scanning control in all modes, presents management, etc. A software system was installed on the standard computer and was developed using Microsoft Visual C++. It was responsible for the interface between the user and the mechanical sliding track. The user enters the number of steps to move the probe holder. After the probe stops, the user can capture the ultrasound image using the computer. The single captured ultrasound image is called B-scan and is in JPEG format. A software system to control the stepper motor was developed using Arduino software. It handled the step movement request from the computer and was responsible for the movement of the probe holder in the sliding track.

\section{B. Volume Reconstruction Formulation}

Transformation of several coordinate systems in homogeneous matrix representation was required to create $3 \mathrm{D}$ volume from sequences of $2 \mathrm{D}$ ultrasound images. At the beginning of the $3 \mathrm{D}$ volume reconstruction formulation, the number of coordinate systems and the number of transformation should be identified. In our proposed device, coordinate system locations and their physical relationships were illustrated in Figure 6(a). All of the coordinate system origins were marked with a red dot. Image plane $\mathrm{P}$ denoted as $\boldsymbol{p}(u, v)$ was the representation of a $2 \mathrm{D}$ ultrasound image or a B-scan. During volume reconstruction, every pixel's location in $\mathrm{P}$ has to be transformed to the reconstruction volume $\mathrm{V}$ in 3D space, denoted as $\boldsymbol{p}^{\prime}\left(x^{\prime}, y^{\prime}, z^{\prime}\right)$. The origin of image plane $\mathrm{P}$ was set to be located at the top-left corner of the image plane. The $x$-axis was in the lateral direction; the $y$-axis was in the same direction with the probe's beam direction and was vertical to the ground, and the $z$-axis was in the elevation direction and was parallel to the probe movement direction. 


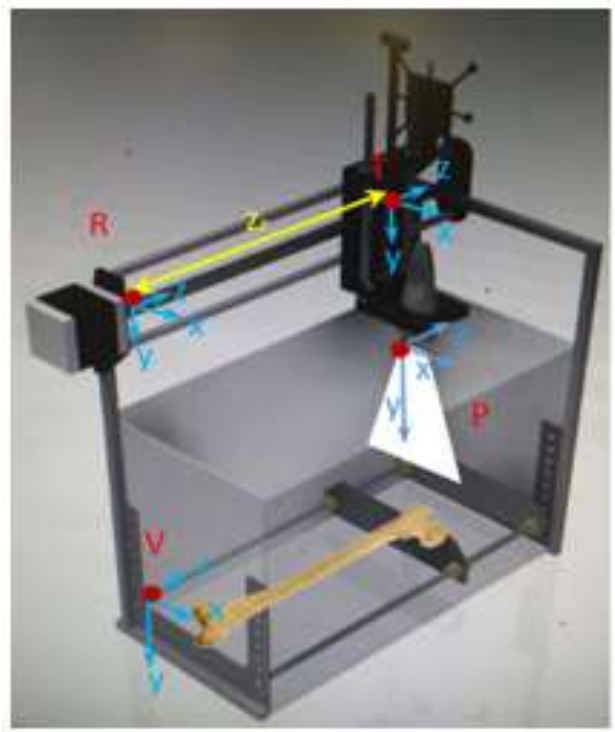

(a)

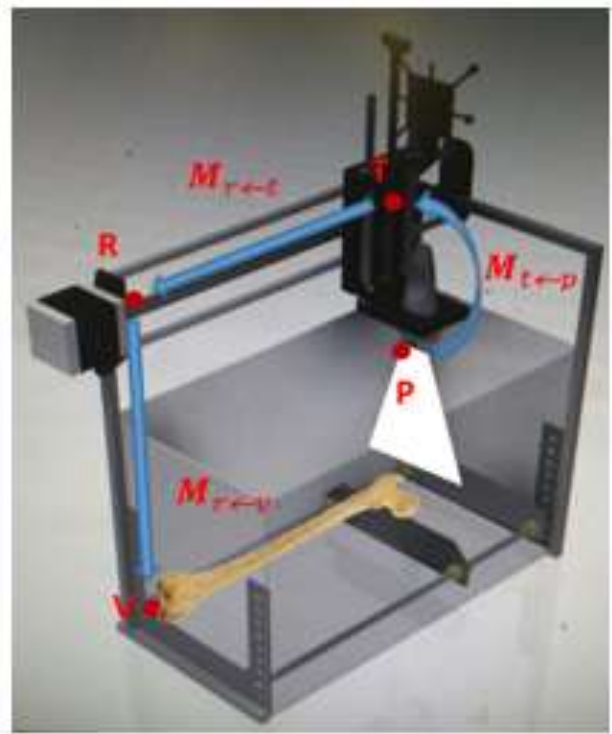

(b)

Figure 6. (a) Identification of the device coordinate systems. P, T, R, and V are the coordinate system of the 2D ultrasound image plane, the probe holder, the origin of the sliding track, and the volume space. (b) Illustration of transformation between P, T, R, and V.

First, each pixel in P was transformed to the probe holder's coordinate system T. The transformation matrix was denoted as $\boldsymbol{M}_{t \leftarrow p}$. The probe holder was fixedly attached to the sliding track. The $x-y$ plane of the coordinate system $\mathrm{T}$ was parallel to the image plane. The next transformation was from the probe holder coordinate system $\mathrm{T}$ to the sliding track $\mathrm{R}$, denoted as $\boldsymbol{M}_{r \leftarrow t}$. Continue with the transformation from the sliding track coordinate system $\mathrm{R}$ to the volume $\mathrm{V}$ denoted as $\boldsymbol{M}_{v \leftarrow t} . \boldsymbol{p}^{\prime}$ denoted as the pixel location in the volume coordinate system $\mathrm{V}$. The homogeneous matrix coordinate system of the image plane $\mathrm{P}$ was $u$ and $v$ as the column and row indices of the B-scan respectively as following:

$$
\boldsymbol{p}(u, v)=\left[\begin{array}{llll}
S_{x} u & S_{y} v & 0 & 1
\end{array}\right]^{T}
$$

where $S_{x}$ and $S_{y}$ were scaling factors in $\mathrm{mm} /$ pixel units. Then the overall transformation from pixel coordinate $\mathrm{P}$ to volume reconstruction coordinate $\mathrm{V}$ was expressed as the homogeneous transformation matrices multiplication as follows:

$$
\boldsymbol{p}^{\prime}=\boldsymbol{M}_{v \leftarrow r} \cdot \boldsymbol{M}_{r \leftarrow t} . \boldsymbol{M}_{t \leftarrow p} \cdot \boldsymbol{p}
$$

The illustration of the overall transformation from pixel coordinate $\mathrm{P}$ to volume reconstruction coordinate V can be seen in Figure 6(b).

Referring to Figure 5, it was known that the position and orientation of the installation of the ultrasound probe could not be ascertained completely perpendicular to the probe holder coordinate system. Therefore, the ultrasound probe position and orientation should be considered in the transformation between $\mathrm{P}$ and $\mathrm{T}$. In this case, probe motion has either translational or rotational joints around all three axes. The transformation from image plane $\mathrm{P}$ to the probe holder $\mathrm{T}, \boldsymbol{M}_{t \leftarrow p}$, comprises of translation of the $x, y$, and $z$, and rotation through angles $\alpha, \beta$, and $\gamma$ around $x$-axis (tilt), $y$-axis (yaw), and $z$-axis (roll) as illustrated in Figure 7(a). It was shown in the picture that an image plane $\mathrm{P}$ could have a particular position and orientation to the origin of the ultrasound probe holder $\mathrm{T}$ depending on the installation of the probe. The illustration of rotational and translational displacement between image plane $\mathrm{P}$ and probe holder $\mathrm{T}$ was shown in Figure 7(b). The coordinate system of $\mathrm{T}$ was illustrated in the dashed red line and the coordinate system of $\mathrm{P}$ was illustrated in the blue line. The mathematical expression of $\boldsymbol{M}_{t \leftarrow p}$ was as follows: 
Tita Karlita, et al.

$$
\boldsymbol{M}_{t \leftarrow p}=\boldsymbol{T}_{t}\left(x_{t}, y_{t}, z_{t}\right) \cdot \boldsymbol{R}_{t}^{z}(\gamma) \cdot \boldsymbol{R}_{t}^{y}(\beta) \cdot \boldsymbol{R}_{t}^{z}(\alpha)
$$

Translational displacement of the $x, y$, and $z$-axis in coordinate system $\mathrm{T}$ was denoted as $\boldsymbol{T}_{\boldsymbol{t}}$

$$
\boldsymbol{T}_{t}\left(x_{t}, y_{t}, z_{t}\right)=\left[\begin{array}{cccc}
1 & 0 & 0 & x_{t} \\
0 & 1 & 0 & y_{t} \\
0 & 0 & 1 & z_{t} \\
0 & 0 & 0 & 1
\end{array}\right]
$$

The rotational displacement of the $x, y$, and $z$-axis with angles of $\alpha, \beta$, and $\gamma$ in coordinate system $\mathrm{T}$ was denoted as $\boldsymbol{R}_{t}^{x}(\alpha), \boldsymbol{R}_{t}^{y}(\beta), \boldsymbol{R}_{t}^{z}(\gamma)$ respectively:

$$
\begin{aligned}
\boldsymbol{R}_{t}^{x}(\alpha) & =\left[\begin{array}{cccc}
1 & 0 & 0 & 0 \\
0 & \cos \alpha & -\sin \alpha & 0 \\
0 & \sin \alpha & \cos \alpha & 0 \\
0 & 0 & 0 & 1
\end{array}\right] \\
\boldsymbol{R}_{t}^{y}(\beta) & =\left[\begin{array}{cccc}
\cos \beta & 0 & \sin \beta & 0 \\
0 & 1 & 0 & 0 \\
-\sin \beta & 0 & \cos \beta & 0 \\
0 & 0 & 0 & 1
\end{array}\right]
\end{aligned}
$$

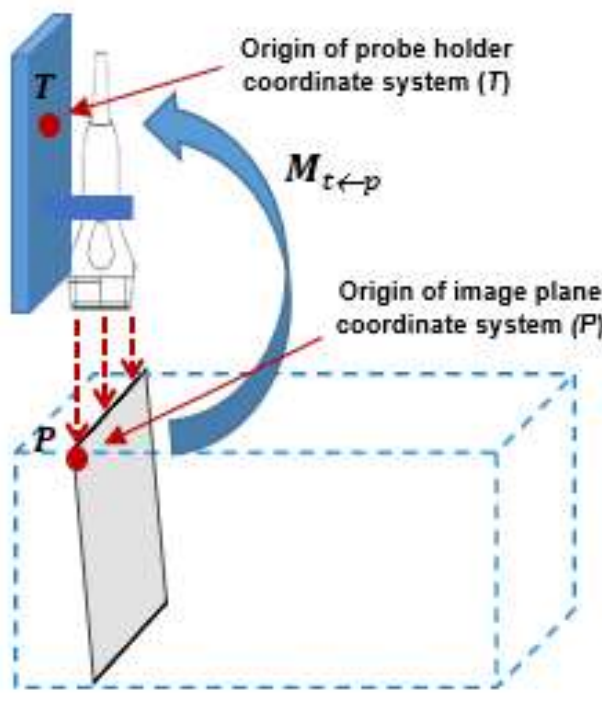

(a)

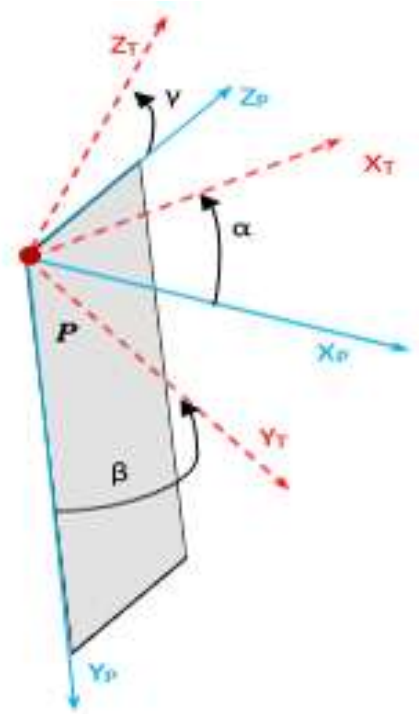

(b)

Figure 7. (a) Illustration of transformation between $\mathrm{P}$ and $\mathrm{T} \boldsymbol{M}_{t \leftarrow p}$. (b) Rotation through angles $\alpha, \beta$, and $\gamma$ around the $x$-axis, $y$-axis, and $z$-axis between image plane $\mathrm{P}$ and probe holder $\mathrm{T}$.

$$
\boldsymbol{R}_{t}^{Z}(\gamma)=\left[\begin{array}{cccc}
\cos \gamma & -\sin \gamma & 0 & 0 \\
\sin \gamma & \cos \gamma & 0 & 0 \\
0 & 0 & 1 & 0 \\
0 & 0 & 0 & 1
\end{array}\right]
$$

Finally, the constrained transformation between image plane $\mathrm{P}$ to the probe holder $\mathrm{T}$ attached to the sliding track, $\boldsymbol{M}_{t \leftarrow p}$, can be represented in the form of

$$
\boldsymbol{M}_{t \leftarrow p}=\left[\begin{array}{cccc}
m_{11} & m_{12} & m_{13} & x_{t} \\
m_{21} & m_{22} & m_{23} & y_{t} \\
m_{31} & m_{32} & m_{33} & z_{t} \\
0 & 0 & 0 & 1
\end{array}\right]
$$

with

$$
m_{11}=\cos \beta \cos \gamma
$$




$$
\begin{aligned}
& m_{12}=\cos \gamma \sin \alpha \sin \beta-\cos \alpha \sin \gamma \\
& m_{13}=\sin \alpha \sin \gamma+\cos \alpha \cos \gamma \sin \beta \\
& m_{21}=\cos \beta \sin \gamma \\
& m_{22}=\cos \alpha \cos \gamma+\sin \alpha \sin \beta \sin \gamma \\
& m_{23}=\cos \alpha \sin \beta \sin \gamma-\cos \gamma \sin \alpha \\
& m_{31}=-\sin \beta \\
& m_{32}=\cos \beta \sin \alpha \\
& m_{33}=\cos \alpha \cos \beta
\end{aligned}
$$

The probe holder $\mathrm{T}$ was fixedly mounted to the linear track and move in one direction along the $z$-axis of the sliding track $\mathrm{R}$. The probe had no rotations and translations movement along with other directions. Therefore, the transformation between these 3D coordinate systems had only 1-DoF. In this formulation, $Z_{r(s t)}$ was denoted as the gap between the origin of $\mathrm{R}$ and the location of the probe at step st. Then, the transformation between $\mathrm{T}$ and $\mathrm{R}, \boldsymbol{M}_{r \leftarrow t}$, can be calculated using Equation 9.

$$
M_{r \leftarrow t}=T_{r}\left(0,0, z_{r(s t)}\right)
$$

Translational displacement of the $z$-axis in coordinate system $\mathrm{R}$ was denoted as $\boldsymbol{T}_{\boldsymbol{r}}$

$$
\boldsymbol{T}_{r}\left(0,0, z_{r(s t)}\right)=\left[\begin{array}{cccc}
1 & 0 & 0 & 0 \\
0 & 1 & 0 & 0 \\
0 & 0 & 1 & z_{r(s t)} \\
0 & 0 & 0 & 1
\end{array}\right]
$$

Assumed that the origin of the sliding track coordinate system $T$ was perpendicular to the origin of volume reconstruction coordinate system $\mathrm{V}$ such that the $z$-axis values of them were the same, then the transformations from R to $\mathrm{V}, \boldsymbol{M}_{v \leftarrow r}$, comprises of translational displacement of the $x$-axis and $y$-axis without any rotation as follows:

$$
\boldsymbol{M}_{v \leftarrow r}=\boldsymbol{T}_{v}\left(x_{v}, y_{v}, 0\right)
$$

Translational displacement of the $x$-axis and $y$-axis in coordinate system $\mathrm{V}$ was denoted as $\boldsymbol{T}_{\boldsymbol{v}}$

$$
\boldsymbol{T}_{v}\left(x_{v}, y_{v}, 0\right)=\left[\begin{array}{cccc}
1 & 0 & 0 & x_{v} \\
0 & 1 & 0 & y_{v} \\
0 & 0 & 1 & 0 \\
0 & 0 & 0 & 1
\end{array}\right]
$$

Finally, using Equation 2 the pixel's location in image plane $\mathrm{P}$ denoted as $\boldsymbol{p}(u, v)$ can be transformed in the $3 \mathrm{D}$ space of volume reconstruction $\mathrm{V}$ denoted as $\boldsymbol{p}\left(x^{\prime}, y^{\prime}, z^{\prime}\right)$. The value of $x^{\prime}, y^{\prime}, z^{\prime}$ in $\mathrm{V}$ can be determined in Equation 13, Equation 14, and Equation 15 respectively.

$$
\begin{aligned}
& x^{\prime}=x_{t}+x_{v}-S_{y} v(\cos (\alpha) \sin (\gamma)-\cos (\gamma) \sin (\alpha) \sin (\beta))+S_{x} u(\cos (\beta) \cos (\gamma)) \\
& y^{\prime}=y_{t}+y_{v}+S_{y} v(\cos (\alpha) \cos (\gamma)+\sin (\alpha) \sin (\beta) \sin (\gamma))+S_{x} u(\cos (\beta) \sin (\gamma)) \\
& z^{\prime}=z_{r(s t)}+z_{t}+S_{y} v(\cos (\beta) \sin (\alpha))-S_{x} u(\sin (\beta))
\end{aligned}
$$

It was noticed that there were six unknown parameters $\left(\alpha, \beta, \gamma, x_{t}, y_{t}\right.$, and $\left.z_{t}\right)$ in $\boldsymbol{M}_{t \leftarrow p}$. In $\boldsymbol{M}_{r \leftarrow t}$, parameter $z_{r(s t)}$ can be measured by running the probe in the sliding track and calculating the distance of probe movement in $z$-axis per step in mm units. Two parameters, $x_{v}$ and $y_{v}$, in $\boldsymbol{M}_{v \leftarrow r}$ were known by manually measure the device dimension. There were eight parameters in total to be identified when $S_{x}$ and $S_{y}$ were taken as scaling factors for the image resolution of the B-scan. In the real application, the calibration process was needed to perform to solve this problem. During the calibration process, a set of nonlinear homogeneous equations was built to determine these parameters. Classical optimization algorithm such as the Levenberg-Marquardt algorithm was an example of a method to solve this nonlinear problem. At the end of the calibration process, the results for translations were in $\mathrm{mm}$, for rotations in degrees, and for scaling factors in $\mathrm{mm} /$ pixel.

\section{Quality Evaluation of Scanning Result}

As stated before, the ultrasound probe's motion and pose (position and orientation) were controlled in our mechanical linear scanning device. Therefore, it was expected to produce homogenous and regularly ultrasound images compared to freehand scanning approach. In 
result, the 3D reconstruction quality of the mechanical linear scanning device should be better than the freehand one. In this case, estimating intrinsic geometric properties of a point cloud is the key factor to judge the quality of both scanning results. The general methods to compare the quality and similarity of the scanning results were performed by measuring the geometric properties quantitatively and the visualization between them [24][25].

To reconstruct the 3D volume, a collection of ultrasound image should be segmented to obtain the object outer contours. In this study, the local phase and first searching method approach for bone outer contour detection method in [2] was applied to generate object outer contours. Then, the object outer contours were arranged systematically (Figure 12) to produce a point cloud in space. A point cloud was characterized as a set of 3D points in space depicting an object's outside surface. Each sample was outlined by its position that was obtained by the measured $X, Y$, and $\mathrm{Z}$ coordinates. When the user enters the same step for each capture of $\mathrm{B}$ scan, then sequentially and regularly spaced $2 \mathrm{D}$ B-scans in $z$-axis will be produced as shown in Figure 8(a). The direction of the $z$-axis was the same as the probe movement direction, and the $y$-axis was in the vertical direction. The illustration of the construction of 3D volume from full sequences of B-scans can be seen in Figure 8(b). Coordinate systems of B-scan and reconstruction volume were represented in pixels and voxels, respectively. All other coordinate systems were represented in $\mathrm{mm}$.

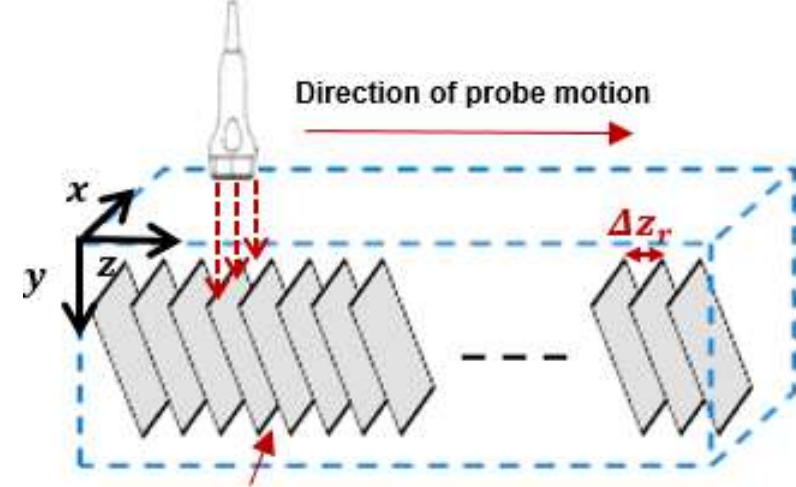

Sequences of regular spaces B-scans

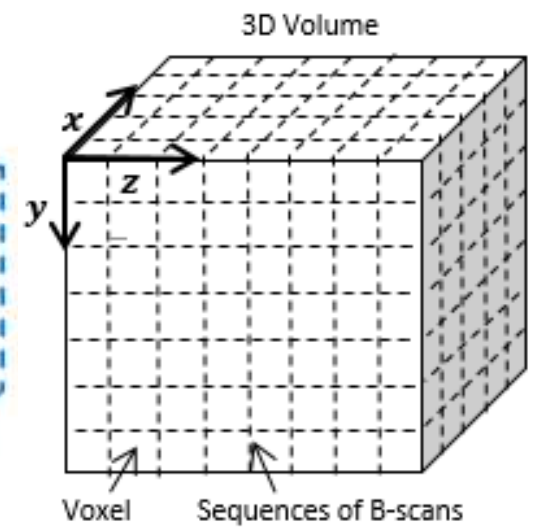

Voxel Sequences of B-scans

Figure 8. (a) Full sequences of B-scans. (b) Construction of sequences of B-scans in the volume coordinates system

To evaluate a point cloud quantitatively, some properties such as density (number of neighbors, surface density, and volume density), curvature, roughness, and distance between two point clouds were taken into account. Moreover, a visual evaluation was performed by constructing meshes from the point clouds.

\section{Density}

A point density in a point cloud was obtained by calculating the number of neighbors $\mathrm{N}$ within a sphere of radius $\mathrm{R}$ for each point. A surface density, $\rho S$, was computed by dividing the number of neighbors $\mathrm{N}$ with the neighborhood surface as formulated in Equation 16. Besides, a volume density, $\rho V$, was obtained by dividing the number of neighbors $\mathrm{N}$ with the neighborhood sphere volume as expressed in Equation 17. The density was calculated for each of $n$ total number of the point clouds, $k=1, \ldots, n$, with the possibility of having a different value of the radius, $\mathrm{R}$.

$$
\begin{aligned}
& \rho S_{k}=\frac{N}{\pi R_{k}^{2}} \\
& \rho V_{k}=\frac{N}{\frac{4}{3} \pi R_{k}^{3}}
\end{aligned}
$$




\section{Curvature and Roughness}

Understanding the curvature of a point cloud gave one a better understanding of the local manifold geometry. In this study differential invariant properties such as Gaussian and mean curvatures were performed since they were one of the essential features in assessing boundarybased geometry. The sign of the curvature of a point clouds can be used to characterize the surface.

Let $p$ be a point on the point cloud $S$. Each plane through $p$ containing the normal line to $S$, cuts $S$ in a plane. A signed curvature which always containing the normal line to that curve can vary as the plane was rotated by an angle $\theta$. Then, the maximal curvature $\kappa_{1}$ and minimal curvature $\kappa_{2}$ were chosen as the principal curvatures of $S$. The mean curvature $H$ at $p \in S$ was calculated as an average of the signed curvature over all angles $\theta$ (Equation 18). By applying Euler's theorem the mean curvature was equal to the average of the maximal curvature $\kappa_{1}$ and minimal curvature $\kappa_{2}$ (Equation 19). More generally, the mean curvature can be defined as Equation 19. Similarly, Gaussian curvature $K$ of a point cloud $S$ was the product of the principal curvatures, $\kappa_{1}$ and $\kappa_{2}$, at the given point (Equation 20). It determined whether a surface was regionally convex (positive) or regionally saddle-shaped (negative) or zero for planes.

$$
\begin{aligned}
& H=\frac{1}{2 \pi} \int_{0}^{2 \pi} \kappa(\theta) d \theta \\
& H=\frac{1}{n}\left(\kappa_{1}+\kappa_{1}\right) \\
& H=\kappa_{1} \kappa_{2}
\end{aligned}
$$

Another one approach to assess the curvature was by computing the normal change rate, or the magnitude of the rate of change of $\mathrm{T}$. Assume that it took a time $\mathrm{s}$ for an object to move along the curve $\mathrm{C}$ with unit speed. The unit tangent vector $\mathrm{T}$ also depended on time. The curvature was then expressed as the normal change rate of $\mathrm{T}$ as in Equation 21.

$$
\kappa=\left\|\frac{d \mathbf{T}}{d s}\right\|
$$

Surface roughness was obtained by measuring the deviations in the direction of the normal vector of a real surface from its ideal form. To obtain the roughness of a point cloud, first neighbors around each point inside a sphere were counted. Afterward, fitted a plane on the neighbors and then computed the distance between the central point and this plane. The roughness here was the deviation from the mean local surface. If some clouds had a lower density, less than 4 points in the sphere, then they should be considered unfit.

\section{Distance}

Equally important, the distance between reference point cloud $M$ and compared point clouds $F$ was expressed as a scalar value using a Euclidean distance denoted as Equation 22. It was a number that describes of how far two point clouds in space were from each other which was determined by finding their nearest neighbors. Each point in both point clouds had some number of $i$-axes, $1, \ldots, j$. The coordinate value of compared and reference point clouds in the $i$-axes were denoted as $f_{i}$ and $m_{i}$.

$$
d_{E}(F, M)=\sqrt{\sum_{i=1}^{j}\left(f_{i}-m_{i}\right)^{2}}
$$

Commonly, reference point cloud $M$ was denser than compared point cloud $F$ so that the estimated distance was acceptable. When the reference point cloud $M$ was not dense enough, the local modeling was taken into account to get a better model of the reference surface and to obtain a better approximation distance. In this study, a mathematical model was fitted on the reference point cloud $M$ to generate local modeling. Then, a reference point cloud $M$ was replaced by this the reference fitting model $M^{\prime}$. Three local modelings were used namely a least square plane, a 2.5D Delaunay's triangulation, or a quadratic height function. When no local model was used, the Hausdorff distance was utilized (Equation 23).

$$
d_{H}(F, M)=\max _{\forall p \in F} d^{F, M}(p)
$$

The nearest neighbor distance to the reference point cloud $M$ was computed for every point $p$ in a compared point cloud $F$, and the distance between them was carried as a scalar vector 
$d^{F, M}(p)$. Two point clouds were close if every point of either point cloud was close to some point of the other set. The maximum distance was calculated as the largest distance between all points in point cloud $F$ and their nearest neighbor in point cloud $M$.

\section{Visual comparison}

Visual comparison of the scanning result was conducted by comparing the meshes generated from the mechanical linear scanning, and freehand scanning [26]. The Delaunay 2.5D triangulation method was applied. The least squares fitting plane was created as a projection plane. Afterward, the corresponding 2D points were triangulated, and the mesh structure was applied to the points in space.

\section{Experiments and Results}

\section{A. The Mechanical Linear 3D Ultrasound Imaging System Configuration}

The realization and configuration of our proposed 3D ultrasound imaging system design in the real environment were illustrated in Figure 9. Water was taken as a medium transmission of ultrasound waves since ultrasound waves tend to reflect when air meets biological tissue. The scanned bone laid on the object holder and immersed in a water tank in a stationary position and just under the transducer. The ultrasound probe position was perpendicular to the bone as an object being scanned. The position and orientation of the ultrasound probe when scanning the bone was shown in the picture.
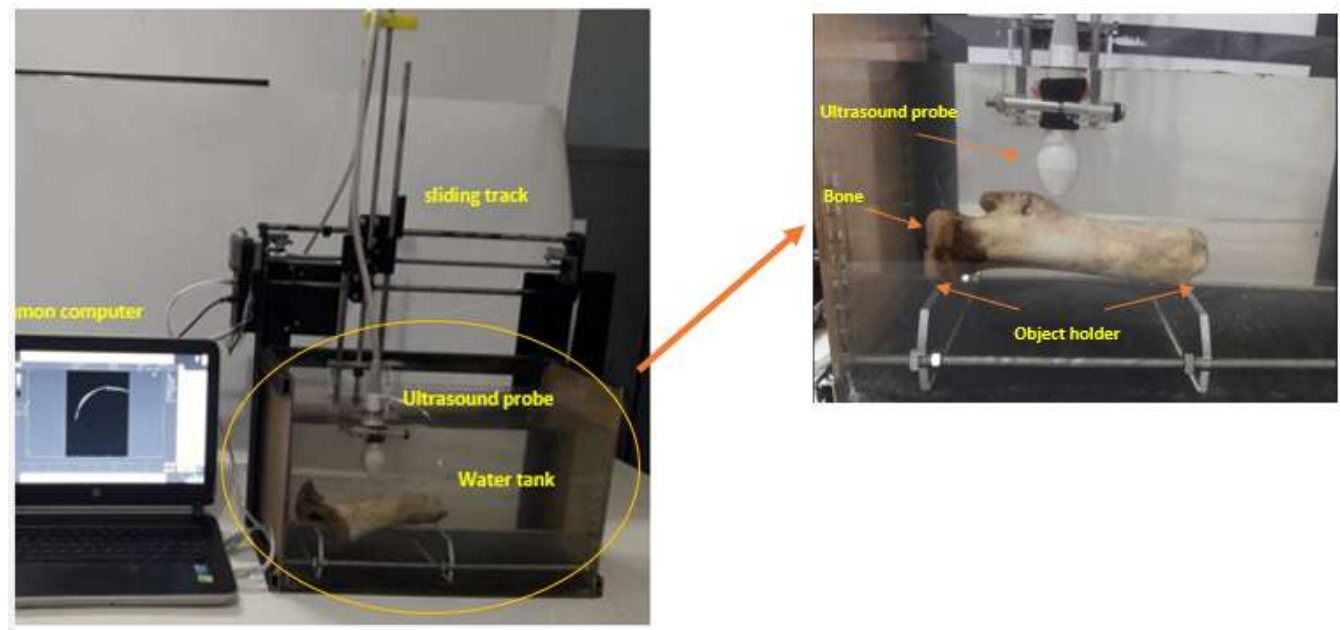

Figure 9. The configuration of the device in the real environment. An ultrasound probe is attached on probe holder running in a linear motion in the sliding track. It is moved by a stepper motor controlled by a software installed on the computer. A bone lying in the water tank and its position against the ultrasound probe in the sliding track.

The bone ultrasound images were then taken by moving probe step by step over the sliding track starting from a starting point (the origin of $V$ ) in the elevation direction along the sliding track. A number of 30 experiments were made to make sure that the probe could move well and to measure the motion and the distance of the probe per step. In each experiment, a various number of steps were taken, and each resulting distance in space was recorded. From the experiments, distance per step of probe movement giving an average number of $6.408333 \times 10^{-3} \mathrm{~mm}$ in the real world was found. By using this calculation as a reference, the amount of step per image capture could be fully controlled by users. The less the step, the denser the B-scan sequence would be. Using this mechanism, a set of B-scans sequentially can be mechanically collected. Figure 10 shows the steps measurement using a caliper. 


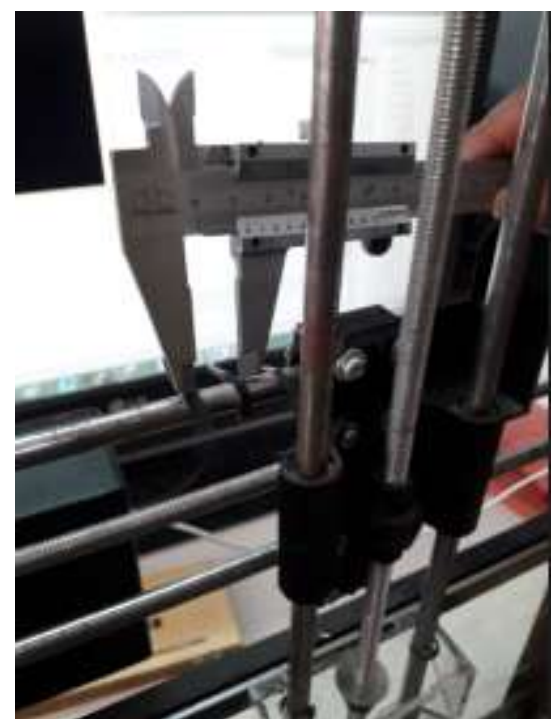

Figure 10. Steps measurement using a caliper to measure the distance per step of probe movement in the sliding track.

\section{B. The Mechanical Linear 3D Ultrasound Imaging System Scanning result}

An example of an ultrasound image as a b-scan produced by the mechanical linear scanning result can be seen in Figure 11(a). $u$ and $v$ indices corresponded with $x$-axes and $y$-axes in 3D space. The position of the B-scans in $z$-axes was acquired by calculating the steps or spacings of $\mathrm{B}$-scan image sequences when the user run the device. To construct the $3 \mathrm{D}$ volume, the full set of B-scans should be arranged sequentially with respect to its positions as shown in Figure 11(b). In the experiment, four sets of ultrasound image were obtained.

As a comparison in assessing the scanning quality of the proposed mechanical linear 3D ultrasound imaging system, we performed a freehand scanning method to the same bone and the same ultrasound probe as in the mechanical linear scanning device. The scanning process is shown in Figure 12; the probe's position was maintained to be perpendicular to the bone which was laid in the water tank. The bone surface was divided into four parts; each part was scanned two or three times starting from one side to the other side. The total number of freehand scanning result was eleven sets. The ultrasound probe was moved by the operator in a steady motion, and the scanning result was saved in avi format with a frame rate 33 per seconds. The bone outer contour of the freehand scanning result was extracted using the [2].
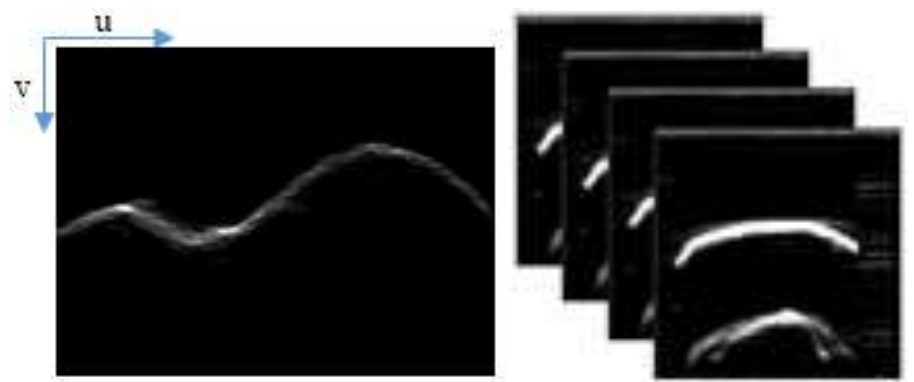

Figure 11. (a) A 2D ultrasound image of the bone. The origin of the image is on the top left corner, (b) the full set of B-scans after arranged sequentially with respect to its positions. 
Tita Karlita, et al.

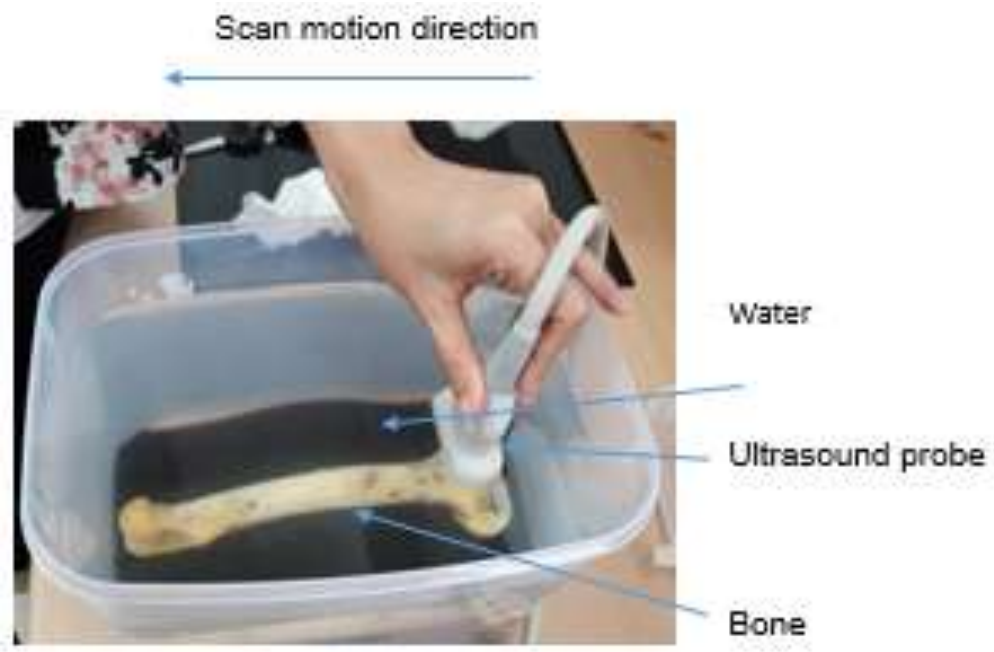

Figure 12. The bone scanning process using freehand techniques. The bone is in steady position under the ultrasound probe.

\section{Point Clouds Construction}

To arrange bone outer contour for the freehand bone point clouds, the gaps between two bone outer contours were determined by dividing the bone length $l$ with the amount of the ultrasound images in one set of scanning process $N$. Therefore, each set of the scanning result might be had a different number of gaps. In Equation 18, $i$ was the sequence number of the scanning result, $i=1, \ldots, 11$.

$$
\operatorname{gaps}_{i}=\frac{l}{N_{i}}
$$

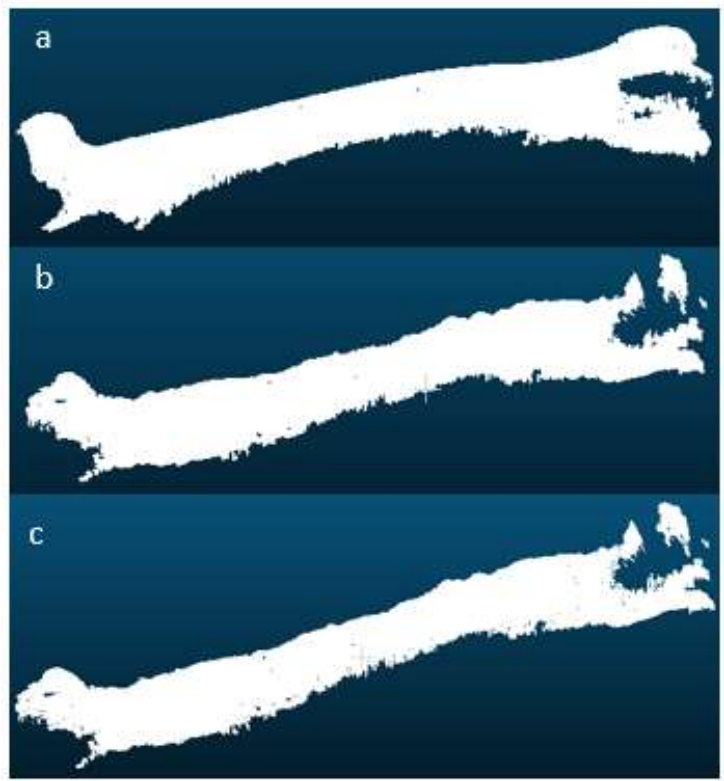

Figure 13. The Samples of point clouds generated from scanning results, (a) from the mechanical linear 3D ultrasound system, (b) from the freehand, and (b) from the freehand after downsampling. 
After segmenting sets of the ultrasound image and extracting their bone outer contours, the point clouds were obtained as shown in Figure 13. In average, the mechanical linear scanning results had around 198.360 points per cloud. While the freehand scanning result had around 343.328 points per cloud. It should be mentioned that in order to get an acceptable quantity comparison; then we sub-sampled each of the point clouds in the freehand scanning approach to decrease the number of points to 198.360 points remaining per cloud. There were no relocations in the original coordinates of the points to maintain the default irregular structure of the content. Point clouds processing in this experiment was done using [27].

\section{Point Clouds Density, Curvature, and Roughness}

The comparison of the relationship of several calculations of point clouds' density, and curvature and roughness are shown in Figure 14. The characteristics values of each point cloud in more detail are described in Table 1 . A radius of $\pm 0.5 \mathrm{~mm}$ was applied on all computation. In this study, the number of neighbors is expressed in points, mean of surface density is in $\mathrm{mm}^{2}$, and mean of volume density is in $\mathrm{mm}^{3}$.
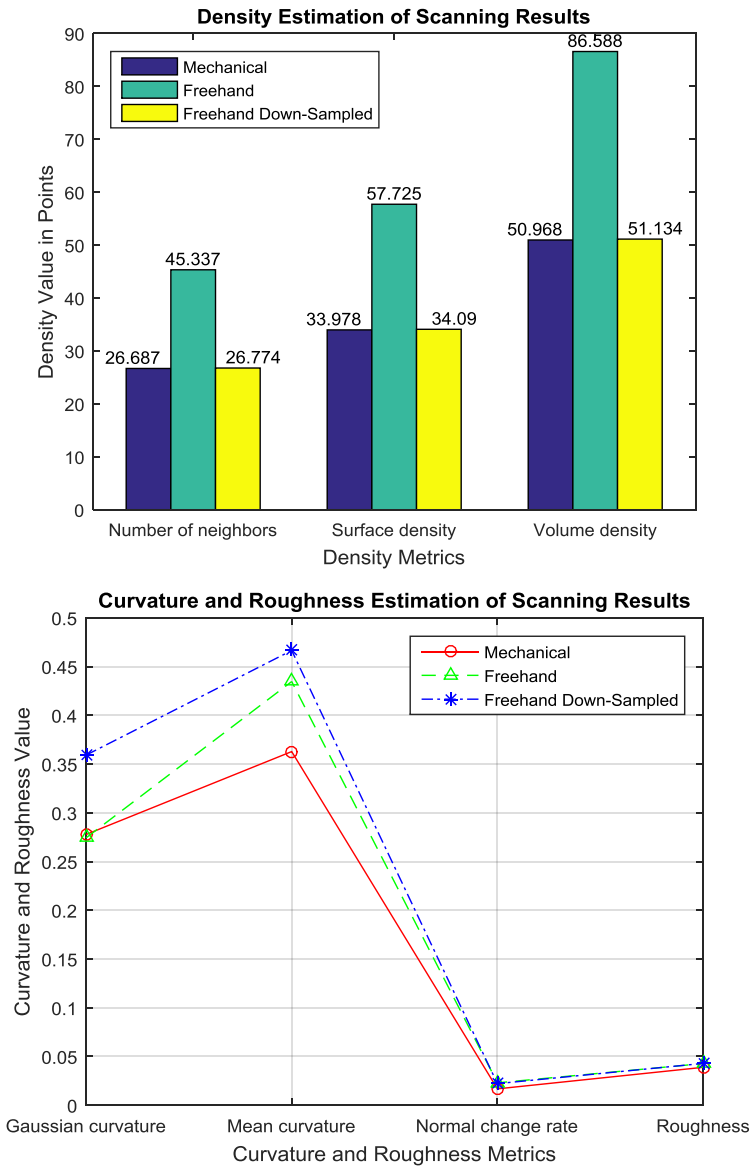

Figure 14. The comparison of point clouds' density, curvature, and roughness between the scanning results of mechanical linear, freehand, and freehand after down-sampled. All computation in a radius of $0.5 \mathrm{~mm}$. 
Table 1. Characteristics of several point clouds' scanning results. The number of neighbors is in points, mean of surface density is in $\mathrm{mm}^{2}$, and mean of volume density is in $\mathrm{mm}^{3}$. All computation use radius of $0.5 \mathrm{~mm}$

\begin{tabular}{|c|c|c|c|c|}
\hline \multicolumn{2}{|c|}{ Properties } & Mechanical & Freehand & Freehand DownSampled \\
\hline \multicolumn{2}{|c|}{ Mean of Number of Points } & 198.360 & 343.328 & 198.618 \\
\hline \multirow{3}{*}{ 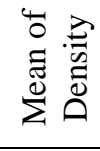 } & Number of neighbors & 26.687 & 45.337 & 26.774 \\
\hline & Surface & 33.978 & 57.725 & 34.090 \\
\hline & Volume & 50.968 & 86.588 & 51.134 \\
\hline \multirow{3}{*}{ 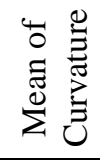 } & Gaussian & 0.278 & 0.274 & 0.359 \\
\hline & Mean & 0.363 & 0.435 & 0.466 \\
\hline & Normal change rate & 0.017 & 0.023 & 0.022 \\
\hline \multicolumn{2}{|c|}{ Mean of Roughness } & 0.039 & 0.043 & 0.043 \\
\hline
\end{tabular}

Figure 15 shows the statistics of three samples of scanning result point cloud (mechanical linear, freehand, and freehand downsampled). All computation of the number of neighbors used radius $0.5 \mathrm{~mm}$. The histogram was a representation of the number of points that have the same value density. Values were represented in small intervals. All histograms are gradually colored from blue to red representing mutual point-to-all-its-neighbors closeness. The red highlighted the strongest, while the dark blue showed the smallest level of those variations. The thin grey curve as a component of every bar graph represented the calculated Gaussian normal distribution of the number of neighbors' distribution. It contained the calculated values of the mean and the standard deviation that was obvious at the top part of each bar. The vertical red line marks the approximate position of each point clouds' number of neighbors complemented with its value and the point count percentage.

Based on Figure 15a, it can be said that the side 1 of the mechanical linear point cloud; which contained 193.142 points in total; more than 77,000 points had a number of neighbors of 44 points in the sphere of radius $0.5 \mathrm{~mm}$. Furthermore, using Gauss distribution fitting, the number of neighbors has an average number of 44.867 and a deviation of 10.537. In the same way, based on Figure 15b, it can be said that the side 1 of the freehand point cloud; which was constructed from 314.685 points in total; more than 130,000 points had a number of neighbors of 28 points in the sphere of radius $0.5 \mathrm{~mm}$. Furthermore, using Gauss distribution fitting, the number of neighbors has an average number of 28.703 and a deviation of 10.537. Furthermore, based on Figure $15 \mathrm{c}$, it can be said that the side 1 of the freehand down-sampled point cloud; which contained 198.618 points in total; more than 84,000 points had a number of neighbors of 26 points in the sphere of radius $0.5 \mathrm{~mm}$. Furthermore, using Gauss distribution fitting, the number of neighbors has an average number of 26.129 and a deviation of 6.715 .

\section{E. Point Clouds' Distance}

Among the outputs of the mechanical linear and the freehand scanning results, there was two bone surface that had a similar surface area. These two pairs of scanning results (mechanical linear scanning result and freehand down-sampled scanning result's version) were used to compare distances between two point clouds. The freehand down-sampled scanning result's version was chosen since it had the relatively same number of points. The comparison of average point clouds' distance, without local model and with several different local models is shown in Figure 16. As stated before, the Hausdorff metric was chosen when no local model used. In the point clouds computation distance, the mechanical linear scanning result acted as reference cloud, and the freehand down sampled acted as a compared cloud. 


\section{F. Visual Comparison}

To compare the quality of the proposed mechanical linear 3D ultrasound imaging system and the freehand scanning results, mesh generation was applied using 2.5D Delaunay triangulation. To enhance the visualization, mesh smoothing using Laplacian method with 20 iterations and 0.2 smoothing factor was applied. The freehand down-sampled scanning result's version were used in this experiment. The result of mesh generation using two similar pair surface of both scanning results can be seen in Figure 17.

\section{Discussions}

A 1-DoF mechanical linear 3D ultrasound imaging system with a new technique of linear mechanical scanning has been proposed and developed. The design of the device has been presented and the usage of the device has been demonstrated in the real environment. The four different coordinates system of our device has been illustrated, and the transformations needed by our system to construct the 3D volume has been analyzed. Finally, the mathematical formulations to transform every pixel's location of the B-scan to 3D volume coordinate system were presented in Equation 13, Equation 14, and Equation 15.
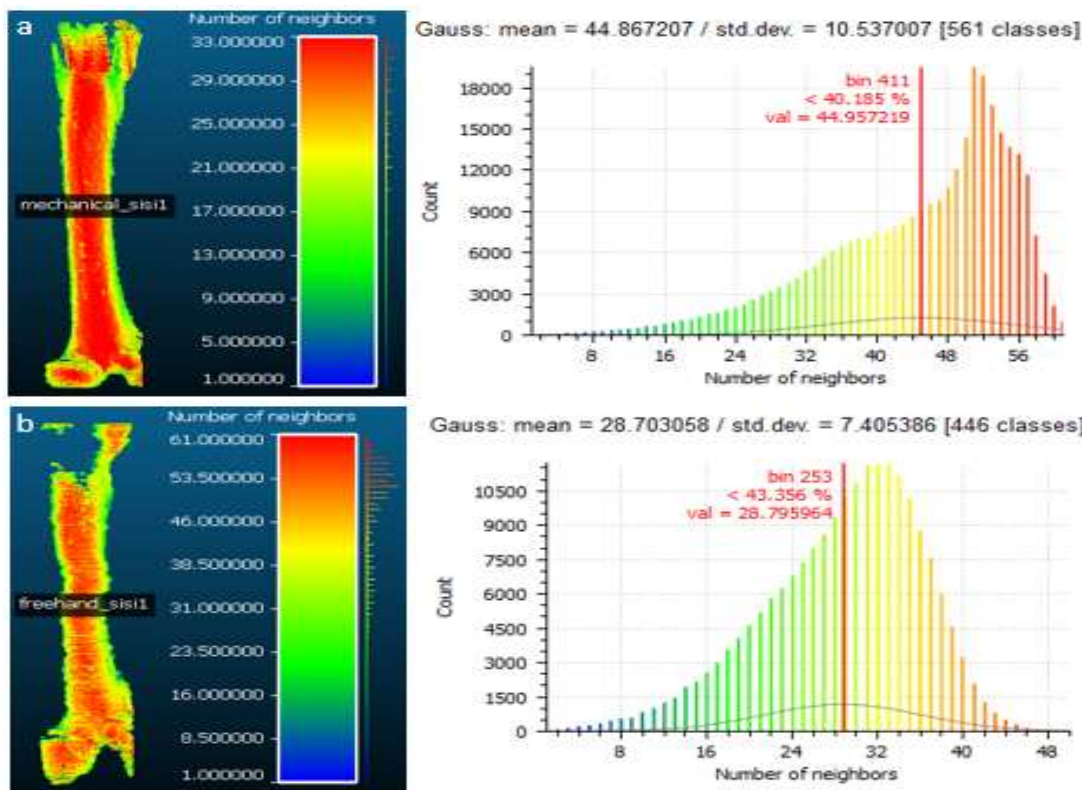

Gauss: mean $=28.703058 /$ std. dev $=7.405386[446$ classes $]$
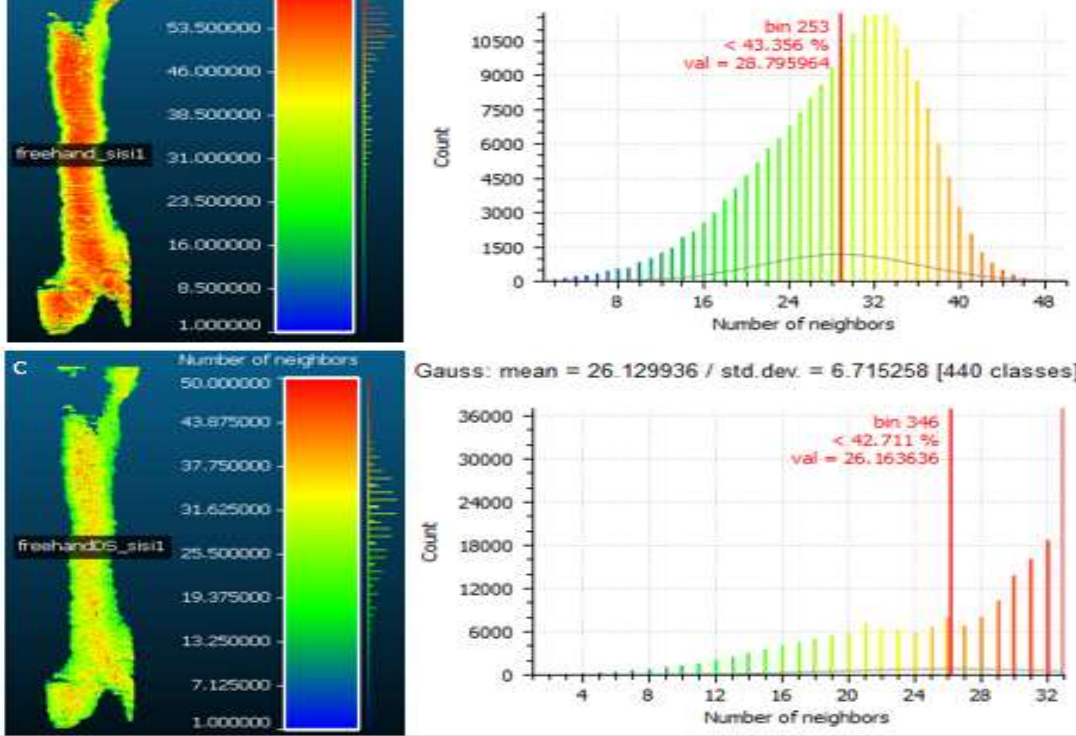

Gauss: mean $=26.129936 /$ std. dev $=6.715258$ [440 classes $]$

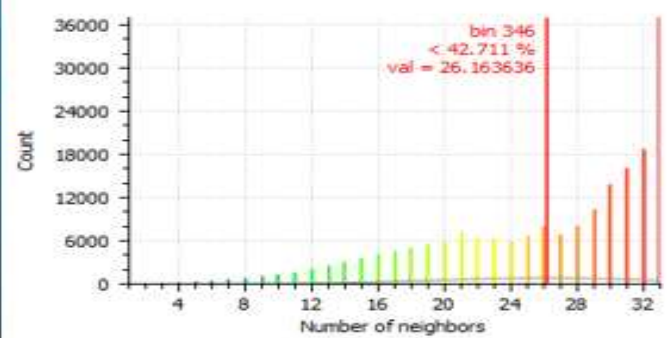

Figure 15. The scalar field diagram of the number of neighbors of one sample of point cloud generated from (a) the mechanical linear 3D ultrasound imaging system (consist of 193.142 points), and (b) the free hand (consist of 314.685 points), and (c) the free hand down-sampled

(consist of 198.618 points) using radius $0.5 \mathrm{~mm}$. On the right side are the corresponding density histogram and the grey curve corresponding to the fitted Gauss distribution. 
However, the DoF reduction of our system led to the limitations of its flexibility. The probe could not move freely in an arbitrary manner during data collection. Despite its limitation, it has some advantages in comparisons with a tracked freehand 3D ultrasound system. The probe was moved in a fix steps depend on the user input during data collection resulting in the regular and densely set of B-scans. The distance of the step can be easily adjusted by the user depending on application requirements. Therefore, it took less effort because the hole filling method to fill empty spaces that require more computation time can be avoided. The components of our system could be found easily in the market at relatively low prices. Our system did not need position sensors, so it did not require special environmental conditions. It was well recognized that the utilization of position sensors in 3D medical imaging systems need special environment settings to ensure the correctness reading of their position and orientation estimation. Data sensing using optical sensor needs a clear line of sight between the marker attached to the probe and the tracking cameras. The electromagnetic sensor is affected by metallic or ferromagnetic materials. Since the scanning path was constrained in one single linear path, there was no rotational error exist in our proposed system when arranging B-scans. The rotational errors are resulting in more significant inaccuracies than the translational error in the reconstruction process. By using our proposed device, the rotational error did not exist and eventually improved the $3 \mathrm{D}$ volume result.

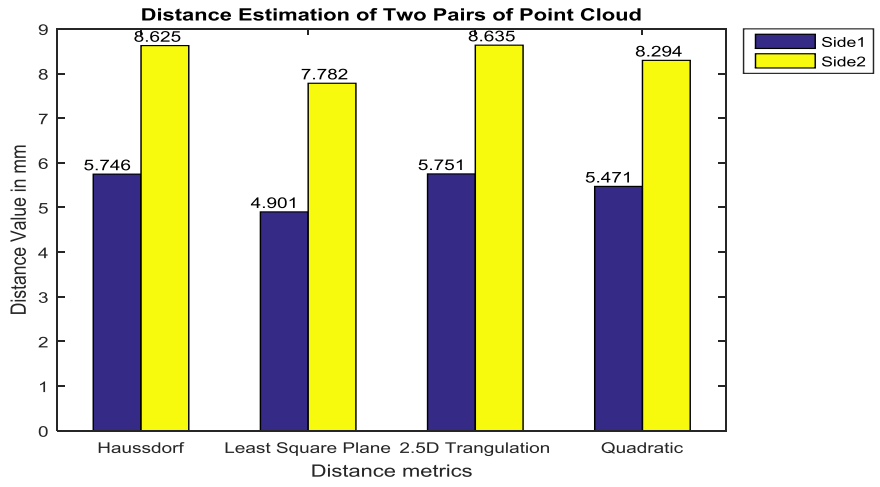

Figure 16. Distance estimation of two pairs (the mechanical linear and the freehand downsampled) of the point cloud using several different distance metrics on two samples of different bone surfaces.

In the experiments, a total of four and eleven bone scanning results was obtained using the mechanical linear 3D ultrasound imaging system and freehand scanning, respectively. In order to compare the scanning results, a downsampling process was conducted to all of both scanning results such that they had a similar point clouds number at the number of 198.618 points per cloud. According to Figure 13, the freehand down-sampled version has a similar point cloud appearance with the original one. This shows that the downsampling process does not significantly change the original shape.

Understanding the curvature and roughness of a point cloud gave one a better understanding of the local manifold geometry. The higher the curvature score, the more elliptical the point cloud will be. Curvature was the amount by which a geometric object deviates from being a flat plane. In Figure 14, the comparison of density, curvature, and roughness of all point clouds produced by the mechanical linear, freehand, and freehand down-sampled was shown. Based on the bar chart, it can be reported that the freehand's point clouds were denser than its down-sampled point clouds and the mechanical linear's point clouds. This was reasonable because the freehand scanning results produced points $173 \%$ more than mechanical linear point clouds. This problem was a matter of the setting parameters in the scanning process. In the mechanical linear scanning setting, the lesser the gap, the denser the point clouds will be. In the freehand scanning setting, the slower the operator moves the probe along the bone, the denser the point clouds will be. Therefore, to have a point cloud that had relatively the same number of point with the mechanical 
linear scanning, the freehand point clouds were downsampled to decrease point's amount without changing the location of the remaining points.
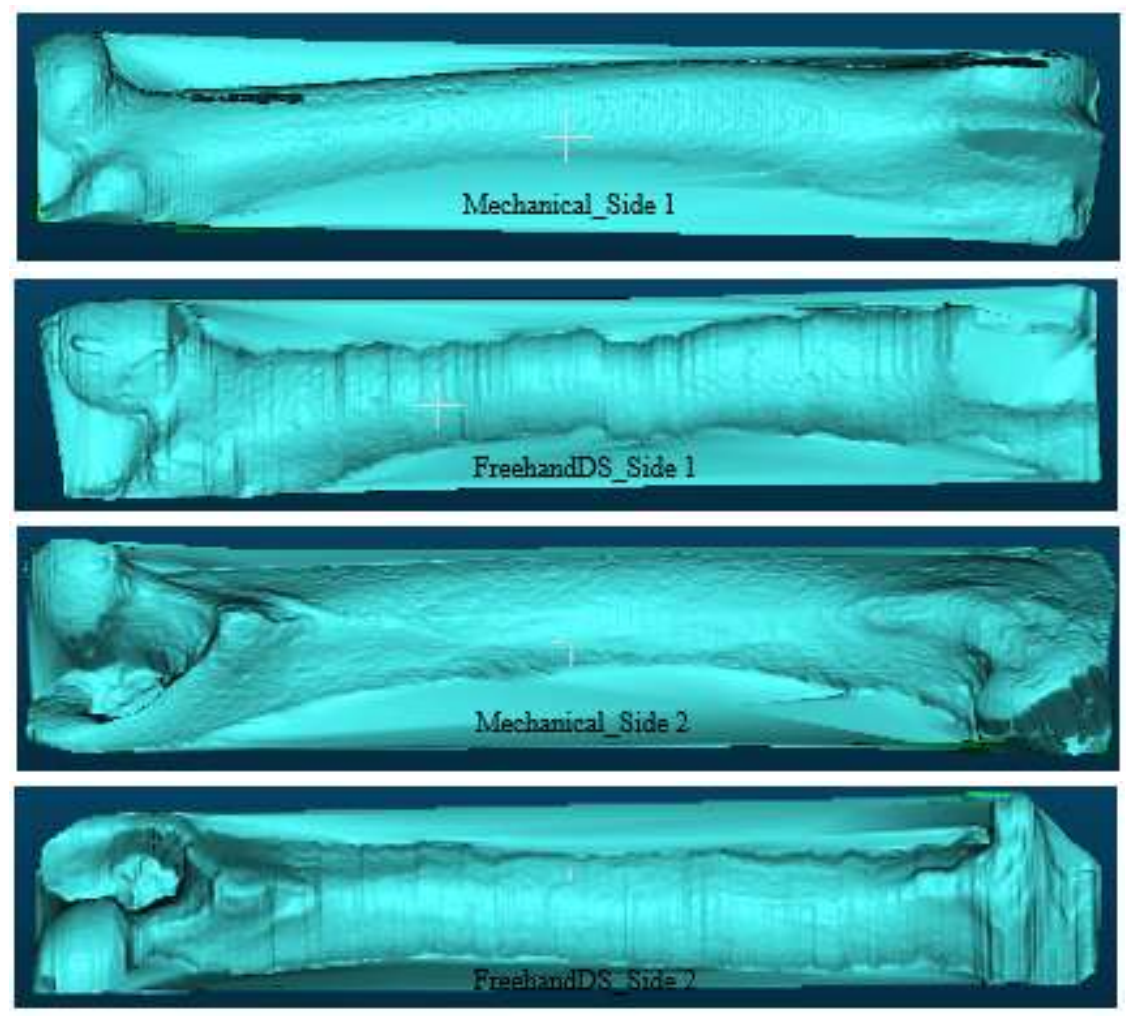

Figure 17. Mesh generation of the proposed mechanical linear 3D ultrasound imaging system and the freehand scanning results using two pairs of similar surface area.

As shown in the line chart in Figure 14 and Table 1, the mechanical linear and the freehand scanning had a similar positive value of Gaussian curvature at around 0.27. On the other hand, the freehand down-sampled had a little higher curvature value at 0.36 . It means that all of the scanning results have an elliptic point. For the mean curvature, the mechanical linear point clouds had the lowest score at 0.363 followed by the freehand and freehand down-sampled at the number of 0.435 and 0.466 , respectively. The mean curvature was more directly equivalent to a radius of curvature. Furthermore, from the picture, it was clear that all the scanning results had almost the same value for the normal change rate at around 0.02 and roughness at about 0.04 . Overall, it was clear that for the curvature, the mechanical linear point clouds had the lowest score followed by the freehand and freehand down-sampled. However, their curvature difference was a little with a deviation of only 0.18 .

More detailed investigation of point cloud density can be obtained by referring to the scalar field diagram and its corresponding histogram in Figure 15. For all type of scanning results, it can be observed that the denser points were at the top area of the bone. Those areas were the bone surface that was perpendicular with the ultrasound probe signal. Nevertheless, the top surface of the freehand downsampled version was less densely than others. Most likely this was due to the effect of the randomize methods of the down sampling process. According to the histogram, it was clear that the number of neighbors distribution of the freehand scanning results followed the normal distribution. Besides, the number of neighbors density of the mechanical linear scanning results tend to lean to the right. It indicated that the mechanical linear point cloud was homogenous densely in space than others. 
The graph in Figure 16 depicts the distance estimation between two point clouds generated from the mechanical linear and the freehand down-sampled scanning results using several different distance metrics as a comparison. According to the graph, the point clouds distance was relatively high at the number of $6.9 \mathrm{~mm}$. If we refer to the surface generation in Figure 17, it can be seen that the mechanical linear's surface was smoother and the surface appearance was more identical and closer to the original shape of the bone phantom than the freehand down-sampled version. In the freehand down sample surfaces, some areas had surface faults or distortions that did not represent the original surface of the bone. Furthermore, it was seen that the mechanical linear scanning results were homogenous and densely such that it can show the detailed structure of the bone surface. Thus, it can be concluded that the mechanical linear scanning results were superior to the freehand version.

\section{Conclusions}

In this paper, a promising alternative of mechanical linear 3D ultrasound imaging system was designed and developed. The usage of our proposed mechanical linear 3D ultrasound imaging system had been demonstrated in the real environment to scan a bone surface. Its scanning results evaluation were provided by generating the point clouds and surfaces. Generally, the system was reliable to create a 3D bone surface in term of quantity and quality results. Finally, it can be concluded that the proposed mechanical linear scanning results were outperformed the freehand version. The device together with $3 \mathrm{D}$ volume reconstruction formulation can be expected will be successfully used to reconstruct $3 \mathrm{D}$ volume and would be useful to clinical applications.

Our future work would determine the overall transformation parameters, i.e., rotation in three directions, translation in three angles, and two scaling factors between the image plane and volume coordinate system. The process of finding these parameters is called calibration. This work will be our future research work. Also, medical image processing and analysis such as registration and surface reconstruction will be included in the proposed system to produce a geometrically accurate $3 \mathrm{D}$ volume.

\section{Acknowledgments}

The research for this paper was financially supported by Program Percepatan Publikasi International (P3I) - Institut Teknologi Sepuluh Nopember, with grant number 011177/IT2.VII/PN.01.00/2018 and Indonesian Endowment Fund for Education (BUDI DNLPDP), Ministry of Finance, Indonesia.

\section{References}

[1]. A. Fenster, D.B. Downey, H.N. Cardinal, Three-dimensional ultrasound imaging, Phys. Med. Biol. 46 (2001) R67-R99.

[2]. T. Karlita, E.M. Yuniarno, I.K.E. Purnama, M.H. Purnomo, Automatic Bone Outer Contour Extraction from B-Modes Ultrasound Images Based on Local Phase Symmetry and Quadratic Polynomial, in: Second Int. Work. Pattern Recognit. (IWPR 2017), Singapore, 2017: pp. 165-170.

[3]. F. Rousseau, P. Hellier, C. Barillot, Robust and automatic calibration method for 3D freehand ultrasound, Med. Image Comput. Comput. Interv. 2003. 9 (2003) 440-448.

[4]. L. Lopez-Perez, J. Lemaitre, A. Alfiansyah, M.-E. Bellemare, Bone Surface Reconstruction Using Localized Freehand Ultrasound Imaging, in: Eng. Med. Biol. Soc. (EMBC), 30th Annu. Int. Conf. IEEE, IEEE, 2008.

[5]. L. Mercier, T. Lang $\varnothing$, F. Lindseth, D.L. Collins, A review of calibration techniques for freehand 3-D ultrasound systems, Ultrasound Med. Biol. 31 (2005) 449-471.

[6]. H. Gao, Q. Huang, X. Xu, X. Li, Wireless and sensorless 3D ultrasound imaging, Neurocomputing. 195 (2016) 159-171.

[7]. H. Xie, K. Hata, A. Manabe, H. Kurioka, K. Takahashi, K. Miyazaki, Three-Dimensional Ultrasonic Volume Measurement of the Ovary in Women With Polycystic Ovary Syndrome, Springer J. Med. Ultrason. 28 (2001) 7-10 
[8]. R.W. Prager, R.N. Rohling, A.H. Gee, L. Berman, Rapid calibration for 3-D freehand ultrasound, Ultrasound Med. Biol. 24 (1998) 855-869

[9]. M.K. Ackerman, A. Cheng, E. Boctor, G. Chirikjian, Online Ultrasound Sensor Calibration Using Gradient Descent on the Euclidean Group, in: IEEE Int. Conf. Robot. Autom., 2014: pp. 4900-4905.

[10]. P.-W. Hsu, R.W. Prager, A.H. Gee, G.M. Treece, Real-time Freehand 3D Ultrasound Calibration, Ultrasound Med. Biol. 34 (2008) 239-251.

[11]. M.M. Baba, O.A. Mohamed, F. Awwad, M.I. Daoud, A Low-Cost Camera-based Transducer Tracking System for Freehand Three-Dimensional Ultrasound, in: New Circuits Syst. Conf. (NEWCAS), 2016 14th IEEE Int., Canada, 2016: pp. 3-6.

[12]. Q.H. Huang, Z. Yang, W. Hu, L.-W. Jin, G. Wei, X. Li, Linear tracking for 3-D medical ultrasound imaging., IEEE Trans. Cybern. 43 (2013) 1747-54.

[13]. R.J. Housden, A.H. Gee, G.M. Treece, R.W. Prager, Sensorless reconstruction of unconstrained freehand 3D ultrasound data, Ultrasound Med. Biol. 1 (2007) 408-419.

[14]. R.-F. Chang, W.-J. Wu, D.-R. Chen, W.-M. Chen, W. Shu, J.-H. Lee, L.-B. Jeng, 3-D US frame positioning using speckle decorrelation and image registration, Ultrasound Med. Biol. 29 (2003) 801-812.

[15]. R.W. Prager, A.H. Gee, G.M. Treece, C.J.C. Cash, Sensorless freehand 3D ultrasound using regression of the echo intensity, Ultrasound Med. Biol. 29 (2003) 437-446.

[16]. A.H. Gee, N.E. Houghton, G.M. Treece, R.W. Prager, 3D ultrasound probe calibration without a position sensor, Ultrasound Med. Biol. (2004) 1-20.

[17]. S. Lessard, I. Bonev, P. Bigras, L.-G. Durand, G. Soulez, G. Cloutier, J.A. De Guise, Parallel Robot for Medical 3D-Ultrasound Imaging, IEEE Int. Symp. Ind. Electron. 4 (2006) 3102-3107.

[18]. P.M.B. Torres, J.M. Sanches, P.J.S. Goncalves, J.M.M. Martins, 3D femur reconstruction using a robotized ultrasound probe, Proc. IEEE RAS EMBS Int. Conf. Biomed. Robot. Biomechatronics. (2012) 884-888.

[19]. P.-W. Hsu, Freehand Three-Dimensional Ultrasound Calibration, A thesis submitted for the degree of Doctor of Philosophy, 2007.

[20]. P.-C. Li, C.-Y. Li, W.-C. Yeh, Tissue Motion and Elevational Speckle Decorrelation in Freehand 3D Ultrasound, Ultrason. Imaging. 24 (2002) 1-12.

[21]. Q.H. Huang, Y.P. Zheng, A new scanning approach for limb extremities using a water bag in freehand 3-D ultrasound, Ultrasound Med. Biol. 31 (2005) 575-583.

[22]. A.H. Gee, G.M. Treece, R.W. Prager, C.J.C. Cash, L. Berman, Rapid registration for wide field of view freehand 3D ultrasound, IEEE Trans. Med. Imaging. 22 (2003) 1344-1357.

[23]. S. Merouche, L. Allard, E. Montagnon, G. Soulez, P. Bigras, G. Cloutier, A robotic ultrasound scanner for automatic vessel tracking and three-dimensional reconstruction of b-mode images, IEEE Trans. Ultrason. Ferroelectr. Freq. Control. 63 (2016) 35-46.

[24]. J. Zhang, W. Huang, X. Zhu, J. Hwang, A Subjective Quality Evaluation for 3D Point Cloud Models, in: ICALIP, 2014: pp. 827-831.

[25]. Javaheri, C. Brites, F. Pereira, J. Ascenso, Subjective and objective quality evaluation of 3D point cloud denoising algorithms, in: IEEE Int. Conf. Multimed. Expo Work., 2017: pp. $1-6$.

[26]. E. Alexiou, T. Ebrahimi, On the performance of metrics to predict quality in point cloud representations, in: Appl. Digit. Image Process. XL. Int. Soc. Opt. Photonics, SPIE, p. $103961 \mathrm{H}$.

[27]. D. Girardeau-Montaut, CloudCompare - Open Source project. 3D point cloud and mesh processing software Open Source Project. 


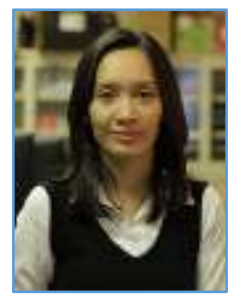

Tita Karlita is a doctoral student at the Department of Electrical Engineering - Institut Teknologi Sepuluh Nopember. She received her S. Kom. and M. Kom. in Department of Computer Science from the Institut Teknologi Sepuluh Nopember, Indonesia in 2002 and 2006, respectively. Her current research interests include $3 \mathrm{D}$ reconstruction, image processing, and computer vision.

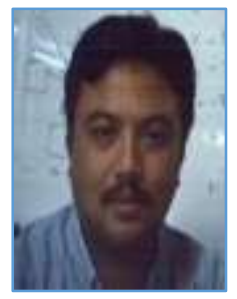

Eko Mulyanto Yuniarno received his S.T. degree in 1994, Master of Technology in 2004, and doctoral degree in 2013 from Department of Electrical Engineering, Institut Teknologi Sepuluh Nopember, Indonesia. Currently, he is a lecturer of Department of Computer Engineering, Institut Teknologi Sepuluh Nopember, Indonesia. His research interest is in image processing, computer vision, and 3D reconstruction.

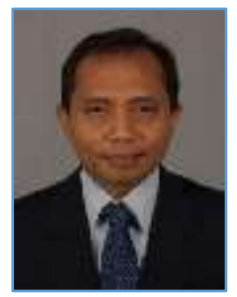

I Ketut Eddy Purnama received his S.T. degree from Department of Electrical Engineering, Institut Teknologi Sepuluh Nopember, Indonesia in 1994. He received Master of Technology degree from Institut Teknologi Bandung, Indonesia in 1999. And he received a doctoral degree from the University of Groningen, The Netherlands in 2007. Currently, he is a lecturer of Department of Computer Engineering, Institut Teknologi Sepuluh Nopember, Indonesia. His research interest is in data mining, medical image processing, and intelligent system.

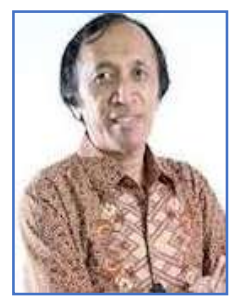

Mauridhi Hery Purnomo received his bachelor degree from Department of Electrical Engineering, Institut Teknologi Sepuluh Nopember, Indonesia in 1985. He received his Master of Engineering and Ph.D. degrees from Osaka City University, Japan in 1995 and 1997 respectively. He joined Institut Teknologi Sepuluh Nopember in 1985 and has been a Professor since 2003. His research interest is in intelligent system application on the electric power system, control and medical signal processing. He is an IAENG and IEEE Member. 